\title{
ENGAGEMENT OF THE LEFT EXTRASTRIATE BODY AREA DURING BODY-PART METAPHOR COMPREHENSION
}

Simon Lacey ${ }^{1}$, Randall Stilla ${ }^{1}$, Gopikrishna Deshpande ${ }^{5,6,7}$, Sinan Zhao ${ }^{5}$, Careese Stephens ${ }^{1}$, Kelly McCormick ${ }^{3}$, David Kemmerer ${ }^{8,9}$, \& K. Sathian ${ }^{1,2,3,4}$ 


\begin{abstract}
Grounded cognition explanations of metaphor comprehension predict activation of sensorimotor cortices relevant to the metaphor's source domain. We tested this prediction for body-part metaphors using functional magnetic resonance imaging while participants heard sentences containing metaphorical or literal references to body parts, and comparable control sentences. Localizer scans identified body-part-specific motor, somatosensory and visual cortical regions. Both subject- and item-wise analyses showed that, relative to control sentences, metaphorical but not literal sentences evoked limb metaphor-specific activity in the left extrastriate body area (EBA), paralleling the EBA's known visual limb-selectivity. The EBA focus exhibited resting-state functional connectivity with ipsilateral semantic processing regions. In some of these regions, the strength of resting-state connectivity correlated with individual preference for verbal processing. Effective connectivity analyses showed that, during metaphor comprehension, activity in some semantic regions drove that in the EBA. These results provide converging evidence for grounding of metaphor processing in domain-specific sensorimotor cortical activity.
\end{abstract}

\title{
KEYWORDS:
}

grounded cognition; embodied cognition; visual cortex; fMRI; resting-state functional connectivity; effective connectivity. 


\section{INTRODUCTION}

The idea that knowledge is represented in abstract codes, distinct from the sensory modalities through which it was acquired (Fodor, 1975), is challenged by theories of grounded cognition. Such theories suggest that knowledge is anchored in modality-specific codes derived from sensorimotor experience and that cognitive processes involve perceptual simulations (Barsalou, 2008). One approach to grounded cognition, conceptual metaphor theory (Lakoff \& Johnson, 1980), argues that knowledge is structured into concepts by metaphorical mappings from sensorimotor experience. For example, when we speak of falling 'behind' schedule or looking 'forward' to an event, we are using our experience of the concrete domain of space to organize and understand the abstract domain of time (Boroditsky, 2000; Casasanto \& Boroditsky, 2008).

These opposing views make very different predictions about the neural basis of metaphor processing. If mapping to sensorimotor experience is involved, then metaphor comprehension should activate brain regions involved in processing the sensorimotor domain from which the metaphor is derived. Consistent with this, texture-selective somatosensory cortex was indeed activated during comprehension of metaphors related to texture (Lacey et al., 2012). By contrast, if sensorimotor mappings are not involved, metaphor processing should involve only classical language regions. Studies reporting such a distribution of activity (e.g., Rapp et al., 2004; Eviatar \& Just, 2006; Lee \& Dapretto, 2006) employed metaphors drawn from a variety of source domains and thus could not properly test the idea of domain-specific sensorimotor cortical recruitment.

A modified version of grounded cognition is suggested by the finding that, as the sense of a word becomes more abstract, neural processing is shifted to the anterior part of the region processing the relevant sensorimotor domain (Chatterjee, 2010; Chen et al., 2008). For example, motion-related metaphors activated the anterior portion of the middle temporal gyrus, a region known to be visually motion-sensitive (Chen et al., 2008). The finding that activation magnitude in motor-related brain regions decreases as the familiarity of 
metaphorical and literal sentences using action verbs increases (Desai et al., 2011) fits with the notion that the perceptual simulations underpinning grounded cognition are weaker or less detailed for more familiar metaphors, reflecting their conventionalization (Bowdle \& Gentner, 2005).

The philosopher-rhetorician Giambattista Vico argued in 1744 that the "greater part of the expressions relating to inanimate things are formed by metaphor from the human body and its parts and from the human senses and passions" (Donoghue, 2014, p89). Body-related metaphors would thus seem ideal to test the different theories of the neural basis of metaphor. Previous imaging studies have concentrated on metaphorical or idiomatic uses of bodyrelated action verbs rather than explicitly named body parts. Action-related metaphors recruit cerebellar and left inferior parietal regions known to be active during movement (Desai et al., 2011, 2013), and left premotor cortex shows body-part congruent activations for action metaphors (Aziz-Zadeh et al., 2006). But while action metaphors elicited somatotopic activation in motor cortex in one study (Boulenger et al., 2009), other studies failed to find activation in motor areas by action metaphors (Raposo et al., 2009) or motion-related idioms, which instead activated a cortical region near visual motion-responsive areas (Chen et al., 2008). However, these studies are limited by the absence of functional localizers (Chen et al., 2008; Desai et al., 2013) or of control sentences matched for meaning (Aziz-Zadeh et al., 2006; Chen et al., 2008; Raposo et al., 2009; Boulenger et al., 2009; Desai et al., 2011, 2013).

Here, we used functional magnetic resonance imaging (fMRI) while participants listened to sentences containing body metaphors referring to faces, arms, or legs. A priori, grounded cognition accounts predict activity at or near several cortical loci during processing of body metaphors, not only in somatosensory and motor cortex, but also in visual cortex, particularly, the face-selective fusiform face area (FFA) (Kanwisher et al., 1997) and the extrastriate body area (EBA). The EBA responds selectively to images of whole bodies and body parts and is located in lateral occipitotemporal cortex (Downing et al., 2001), in the 
inferior temporal sulcus/middle temporal gyrus (ITS/MTG: Myers \& Sowden, 2008; Taylor et al., 2007; Weiner \& Grill-Spector, 2011). Participants heard sentences using body-part words used in either metaphorical or literal contexts, with control sentences matched for meaning and also matched on lexical, semantic, and acoustic variables. In separate sessions, they underwent motor, somatosensory, and visual functional localizer scans. This design aimed to isolate the brain regions underlying both metaphorical and literal use of body-part words, and to determine whether they were in or near the visual, somatosensory or motor regions identified by the localizers. Thus, we could test the grounded cognition hypothesis that comprehension of body-part metaphors should engage sensorimotor cortices involved in processing body parts. Even though this hypothesis makes no specific prediction as to whether body-part metaphor comprehension is grounded in visual, somatosensory, or motor experience, our design covered all the possibilities. In addition, we collected resting-state fMRI data to examine whether body-selective areas active during metaphor processing exhibit functional connectivity with language-related areas and how this relates to individual preferences for visual imagery and verbal processing. Finally, we also carried out effective connectivity analysis of task-state fMRI data, in order to gain converging evidence with respect to the neural basis of metaphor processing.

\section{METHODS}

\subsection{Participants}

Twelve people (six male, six female; mean age 23 years 6 months) took part in the main imaging experiment of this study. All were right-handed based on the validated subset of the Edinburgh handedness inventory (Raczkowski et al., 1974) and none had taken part in the metaphor selection process described below. We excluded volunteers for whom American English was a second or non-native language in order to avoid potential confounds due to variable language ability. All participants gave informed consent and all procedures were approved by the Emory University Institutional Review Board.

\subsection{Metaphor selection}


We compiled a list of sentences containing body-part metaphors via deliberation, internet searches, and creating novel metaphors. We also compiled control sentences that matched the metaphorical ones for meaning. Wherever possible we minimized syntactic differences between metaphorical and control sentences (see below). A schematic of the stimulus generation and preparation process is provided in Figure 1a.

The metaphors were restricted to those related to arms, legs and faces (or sub-parts thereof) because these body parts have readily identifiable cortical associations in motor, somatosensory, and visual cortex. The list was further constrained by the need for all of the sentences to be relatively short and, ideally, for each control sentence to be formed by substitution of a single word - e.g., by changing 'she shouldered responsibility' to 'she took responsibility' - in order to minimize syntactic differences, which was possible in approximately $75 \%$ of the initial list. In the remainder, the metaphor consisted of a phrase and the control sentence contained a substituted, non-metaphorical phrase - e.g., 'she turned a blind eye' vs. 'she took no notice'. This exercise resulted in 69 metaphor-control pairs. We analyzed the numbers of words and syllables in each sentence, and the frequency and imageability of the operative word in each sentence (e.g., 'he had to foot the bill' vs. 'he had to pay the bill'), using the MRC Psycholinguistic Database 2.0 (Coltheart, 1981; Wilson, 1988) and cross-checking with published ratings (Gilhooly \& Logie, 1980; Kucera \& Francis, 1967; Toglia \& Battig, 1978). We assumed a frequency of 1 for any word without a published frequency and conducted our own imageability ratings for words without a published rating, using instructions adapted from Toglia \& Battig (1978). After making adjustments to reduce differences between metaphorical and control sentences for these linguistic variables, we tested the interpretability of the 69 metaphorical sentences, as outlined below, before selecting the final list.

Ten participants read each metaphorical sentence and provided their own interpretation of its meaning. Three independent judges read each participant's interpretations of each metaphorical sentence and rated them as plausible or not (regardless of the intended control 
sentence). We then calculated an interpretability score for each metaphorical sentence as the number of judges who rated an interpretation as plausible for each participant; thus the maximum score for each metaphorical sentence was 30 (10 participants x 3 judges). A cut-off score of $21(70 \%)$ gave 60 metaphorical sentences that were deemed easily interpretable. Of these, 23 related to the arm, 20 to the head, and 17 to the leg. (In order to have equal numbers for each body part, we dropped the arm metaphorical sentences with the three lowest interpretability scores and, in the eventual fMRI experiment, repeated 3 of the leg metaphorical sentences in separate runs - see below). We then assessed how closely the participants' interpretations agreed with the proposed control sentence; a simple count gave 85.8\% agreement. For one metaphorical sentence ('she kept her head down'), no participants' interpretation agreed with the proposed control sentence ('she worked hard') but all participants' interpretations agreed with each other (variants of 'she lacked confidence') and so the control sentence was replaced accordingly. This gave $86 \%$ agreement between metaphorical and control sentences.

\subsubsection{Lexical analyses}

Before finalizing the metaphorical and control sentences, we made further adjustments to the numbers of words and syllables in each sentence, and the frequency and imageability of the operative word in each sentence, in order to balance these variables across sentence types. On average, metaphorical sentences had slightly, but significantly, more words than control sentences, but the sentences did not differ significantly in the number of syllables. There was no significant difference in word frequency but the operative words in the metaphor sentences were more imageable than those in the control sentences (Table 1a).

\subsubsection{Semantic ratings}

Ten participants ( 5 male, 5 female, who were not involved in the initial interpretation exercise) read all the metaphor sentences and their controls and rated them for interpretability (how easy they were to understand), naturalness (whether they seemed normal or forced/unusual), figurativeness (how metaphorical each sentence was), familiarity, 
imageability (how easily the sentence as a whole evoked a mental image), and valence (whether the meaning was positive, negative, or neutral). They also rated the aptness (how suitable each metaphor was to express the intended meaning) of each metaphor and the agreement of each metaphor-control pair (how well the pair matched for meaning). All ratings were made on a 7-point Likert scale. Compared to their control sentences, metaphor sentences were, perhaps unsurprisingly, rated as less interpretable, natural and familiar but, as expected, were considered more figurative and, again unsurprisingly, as more imageable (Table 1b). Judged on their own against the mid-point of the Likert scale however, the metaphorical sentences were rated as highly interpretable, natural, figurative, familiar, imageable and apt (Table 1b), i.e., although metaphors were judged less interpretable, natural, and familiar than their controls, they were not inherently difficult to understand, unnatural, or unfamiliar. We tested against the mid-point because this represents a point at which there is no clear bias to either end of the scale and therefore serves as a reference point to give meaning to the observed ratings (Lacey et al., 2011a). Agreement for meaning between metaphorical and control sentences was also highly rated (Table 1b). We used the valence ratings as the standard against which we assessed the accuracy of participants' responses in the scanner: participants were asked to judge sentence valence with the goal of ensuring deep semantic processing with a task orthogonal to the experimental task. Since the valence judgments are somewhat subjective, we did not place much weight on these ratings other than as a measure of task compliance in the scanner.

\subsubsection{Recording and acoustic analysis}

One author (RS) was recorded speaking the metaphorical and control sentences with minimal inflection. Digital recordings were made in Audacity ${ }^{\circledR}$ v2.0.1 (Audacity Team, 2012) using a SHURE 5115D microphone and an EMU 0202 USB external sound card at a $44.1 \mathrm{kHz}$ sampling rate. In order to reduce fluctuations in ambient noise, equipment characteristics, and the speaker's voice, recordings were made in long 'takes' of 20 sentences. These recordings were then processed in Audacity, using standard tools and default settings, for sound amplification; removal of noise (e.g., hiss), 'clicks and pops'; and finally, high- and 
low-pass filtering to remove ambient noise (e.g., air-conditioning hum). The processed files were segmented into individual sentence files in Audacity and saved as '.wav' files which were subsequently analyzed acoustically using PRAAT (Boersma \& Weenink, 2008) to measure sentence duration (from which speech rate in syllables per second could be derived), mean pitch $(\mathrm{Hz})$ and mean intensity $(\mathrm{dB})$. Metaphorical sentences were slightly, but significantly, longer than control sentences, but there were no significant differences in speech rate, mean pitch, or mean intensity (Table 1c).

\subsection{Literal sentences}

We also created a set of 60 sentences in which body parts (again, arms, legs and faces, or sub-parts thereof) were used in a literal context, along with control sentences in which the body part word was replaced (e.g., 'he washed his face - he washed his car'). These were subject to the same lexical, semantic (except for aptness and agreement which are not relevant to the literal sentences), and acoustic analyses as described above. They were recorded in the same way and by the same person. There were no significant differences between the literal sentences and their controls on any of the variables tested except that literal sentences were considered slightly more interpretable than their controls (Table 2). Examples of metaphorical and literal sentences and their respective control sentences are shown in Table 3.

\subsection{Main experiment procedures}

\subsubsection{Organization of scan sessions}

Three separate scan sessions were conducted (a schematic timeline of the study sequence is provided in Figure 1b). To minimize participant fatigue, the first two, longer sessions were each split into two parts with an intervening break during which participants were brought out of the scanner and allowed to walk about for a few minutes before being repositioned. A structural scan was acquired at the end of each half of the first two sessions and at the end of the last session (five structural scans in total). The first session began with a single, eightminute run of resting-state fMRI data acquisition. We began with this run in order to avoid 
priming of resting-state data by the tasks we used. Following this run, participants were presented with the metaphorical and their control sentences. After a break, the literal and their control sentences were presented - this order was intended to avoid potential priming of processing of metaphors by the literal sentences. The second and third sessions comprised visual, somatosensory, and motor localizer tasks designed to activate the relevant body-part selective cortical areas. In the second session, two of the localizer tasks were presented with an intervening break; the third localizer was administered in the last session. The language session preceded the localizers in order to avoid potential priming of language processing by the sensorimotor localizer tasks. The order of the localizer tasks, and the order of runs within all tasks, was counterbalanced. Presentation software (Neurobehavioral Systems Inc., Albany CA) was used to present visual and auditory stimuli and cues, and to synchronize their presentation with scan acquisition (see below).

\subsubsection{Language tasks}

There were two runs with metaphorical sentences and two runs with literal sentences. Each run lasted 633.6s and contained 30 metaphorical or literal sentences and their associated control sentences, for a total of 60 trials in each condition. Nominal trial length was $2.4 \mathrm{~s}$; the sentences varied in length but all fitted within the $2.4 \mathrm{~s}$ timeframe set as the time in which a single brain volume was acquired in the scanner (see 'MR scanning' below). There was a blank inter-trial interval (ITI) of 7.2s. Each run also contained six pseudorandomly interspersed blank periods of 9.6s to allow for baseline estimation. Each run contained 10 metaphorical or literal sentences and corresponding control sentences for each of the three body parts, i.e. face, arm, and leg, in pseudorandom order. Because there were only 17 leg metaphors, we divided these between the metaphor runs: 9 in one and 8 in the other. In order to have 10 different leg metaphors in each run, we repeated two items from the first run in the second and one from the second run in the first. In this way, the three metaphors which it was necessary to repeat (in order to equalize the number of trials across body parts) did not

repeat within a run. To avoid possible repetition suppression effects resulting from consecutive sentences relating to the same body part, sentences referring to a given body part 
were separated by at least two trials of different body-part sentences and/or control sentences. Participants performed a valence task in which they were asked to judge whether each sentence denoted something positive, negative, or neutral, and to press the appropriate button on a response box using their left hand. This task was designed to promote deep semantic processing of each sentence. Responses were recorded by the Presentation software so that task accuracy and response times (RTs) could be computed; RTs were calculated from the end of each sentence and responses before that time were discarded.

\subsubsection{Visual localizer task}

There were four visual localizer runs, each lasting $751.2 \mathrm{~s}$ and containing 18 trials each for faces, arms, legs and objects, for a total of 72 trials in each condition. The localizer task was modeled on that in Bracci et al. $(2010,2012)$ and utilized some of the images of arms, legs and objects from these studies; face images were derived from the Glasgow Face Matching Test (Burton et al., 2010; downloaded from http://homepages.abdn.ac.uk/m.burton/pages/gfmt). Nominal trial duration was $2.4 \mathrm{~s}$, with presentation of the visual stimulus for $1 \mathrm{~s}$ and $1.4 \mathrm{~s}$ for response. ITI was $7.2 \mathrm{~s}$ and there were five baseline periods of $12 \mathrm{~s}$. As above, trials of the same body part were separated by at least two trials of different body parts and/or objects. Each run contained a single repeated stimulus in each condition, at random intervals, and participants had to press a response button when they detected these.

\subsubsection{Motor and somatosensory localizer tasks}

There were four runs of each task, lasting 633.6s each and containing 20 trials each for the face, arm and leg for a total of 80 trials in each condition. Trial duration was $2.4 \mathrm{~s}$, ITI was $7.2 \mathrm{~s}$ and there were six baseline periods of $9.6 \mathrm{~s}$. Trials of the same body part were separated by two trials of different body parts (there were no control conditions). For the motor localizer, participants responded to auditory prompts with a right hemifacial 'grin', flexion of the fingers of the right hand (without contact between fingertips and palm), or right ankle plantar flexion. For the somatosensory localizer, an experimenter standing at the scanner bore, and cued by visual prompts projected onto the scanner bore, used a foam-rubber 
paintbrush to stroke the right cheek, the right palm, or the sole of the right foot, three times in quick succession within a trial.

\subsection{MR scanning}

MR scans were performed on a 3 Tesla Siemens Trio TIM whole body scanner (Siemens Medical Solutions, Malvern, PA), using a 12-channel head coil. T2*-weighted functional images were acquired using a single-shot, gradient-recalled, echoplanar imaging (EPI) sequence for BOLD contrast. For all functional scans including task and resting-state scans, 39 axial slices of $3.1 \mathrm{~mm}$ thickness were acquired using the following parameters: repetition time (TR) 2400ms, echo time (TE) 30ms, field of view (FOV) 200mm, flip angle (FA) $90^{\circ}$, in-plane resolution $3.125 \times 3.125 \mathrm{~mm}$, and in-plane matrix $64 \times 64$. High-resolution $3 \mathrm{D}$ anatomic images were acquired (five in total, see Section 2.4.1) using an MPRAGE sequence (TR $2300 \mathrm{~ms}$, TE $3.9 \mathrm{~ms}$, inversion time $1100 \mathrm{~ms}$, FA $8^{\circ}$ ) comprising 176 sagittal slices of $1 \mathrm{~mm}$ thickness (FOV 256mm, in-plane resolution $1 \times 1 \mathrm{~mm}$, in-plane matrix $256 \times 256)$. Once magnetic stabilization was achieved in each run, the scanner triggered the computer running Presentation software so that the sequence of experimental trials was synchronized with scan acquisition.

\subsection{Post-scan ratings}

Approximately a month after the end of the experiment, we asked participants to provide familiarity and imageability ratings for the metaphorical and literal sentences so that we could conduct correlation analyses using participants' ratings in relation to their fMRI data. This was carried out in the same way as the semantic ratings described above.

\subsection{Image processing and analysis of activations}

Image processing and analysis was performed using BrainVoyager QX v2.8.2-2.8.4 (Brain Innovation, Maastricht, Netherlands). In individual analyses, each participant's functional runs were real-time motion-corrected utilizing Siemens 3D-PACE (prospective acquisition motion correction). Functional images were preprocessed utilizing cubic spline interpolation 
for slice scan time correction, trilinear-sinc interpolation for intra-session alignment of functional volumes, and high-pass temporal filtering to 2 cycles per run to remove slow drifts in the data without affecting task-related effects. Anatomic 3D images were averaged, coregistered with the functional data, and transformed into Talairach space (Talairach \& Tournoux, 1988).

For group analyses, the transformed data were spatially smoothed with an isotropic Gaussian kernel (full-width half-maximum $4 \mathrm{~mm}$ ). The $4 \mathrm{~mm}$ filter is within the $3-6 \mathrm{~mm}$ range recommended to reduce the possibility of blurring together activations that are in fact anatomically and/or functionally distinct (White et al., 2001). Runs were percent signal change normalized (i.e., the mean signal value for each voxel's time course was transformed to a value of 100 , so that the individual values fluctuated around that mean as percent signal deviations). For statistical analyses, we created a group average brain mask by averaging participants' Talairach-normalized 3D structural brain volumes into a single 3D volume with $1 \mathrm{~mm}$ isotropic spatial resolution, which was then transformed into a 'mask' file with $3 \mathrm{~mm}$ spatial resolution, equivalent to the spatial resolution of the functional data files. For the purpose of displaying activations, we created a group average brain differently in order to minimize spatial distortion. We first selected a representative (target) Talairach-normalized brain from the 12participant group. We then individually aligned the 11 remaining participants' Talairachnormalized brains to this target (co-registration to match the gyral and sulcal pattern, followed by sinc interpolation). These 11 aligned brains were then averaged. This 11-subject average brain was then averaged with the target brain, creating a single Talairach template, with $1 \mathrm{~mm}$ isotropic resolution, which was used to display the activations for the 12-subject group.

Statistical analyses of group data used general linear models (GLMs) treating participant as a random factor (so that the degrees of freedom equal n-1, i.e. 11), followed by pairwise contrasts. This analysis allows generalization to untested individuals. Correction for multiple comparisons within the whole brain (corrected $\mathrm{p}<.05$ ) was achieved by imposing a threshold for the volume of clusters comprising contiguous voxels that passed a voxel-wise threshold of 
$\mathrm{p}<0.05$, using a 3D extension (implemented in BrainVoyager QX) of the 2D Monte Carlo simulation procedure described by Forman et al. (1995). Exceptions to this procedure (more stringent thresholds for some of the localizer tasks) are noted in the Results. Activations were localized with respect to 3D cortical anatomy with the help of an MRI atlas (Duvernoy, 1999). In order to compare activity associated with body part metaphor processing with that relating to body literal sentences, we carried out an interaction contrast, i.e. (metaphor $>$ metaphor control) $>$ (literal $>$ literal control), followed by Bonferroni-corrected post-hoc comparisons (see Nieuwenhuis et al., 2011).

We also conducted analyses treating item as a random factor, thus allowing generalization to untested body-part metaphors (Clark, 1973). Activations present on both participant-based and item-based analyses would enable us to conclude that the results generalize beyond both the specific participants scanned and the specific body-part metaphors used (Bedny et al., 2007; Lacey et al., 2011a). Following a procedure described previously (Bedny et al., 2007; Lacey et al., 2011a), all sentences were itemized so that each trial, excluding the 3 repeated leg metaphors and their controls, was identified as a discrete condition (metaphor 1, metaphor $2 \ldots$ metaphor 57; metaphor control 1, metaphor control $2 \ldots$ metaphor control 57). Thus each metaphor could be paired to its appropriate control. Standardized beta maps were created for each item, z-transformed and averaged across participants, and used to construct a GLM with item as a random factor. We then specified itemized pair (metaphor $>$ metaphor control) beta maps and performed a 1-sample t-test on these differences, equivalent to carrying out a paired t-test on data averaged across subjects for each item. Before carrying out these item-pair analyses, we used the ANCOVA option in BrainVoyager to filter out activity related to acoustic nuisance variables (mean pitch, mean intensity and sentence duration) which are unrelated to metaphor processing.

We did not include the semantic variables (metaphor familiarity and imageability) in this ANCOVA because they are intimately related to metaphor processing in grounded cognition accounts. As set out in the Introduction, perceptual simulations are thought to underlie the 
grounding of cognition in sensorimotor experience, and visual imagery is a prime example of such simulations (Barsalou, 2008) - therefore the imageability of the metaphor is potentially important, and may modulate activation magnitude as the ease of imagery varies. These simulations may be more weakly activated, or be less detailed, as a metaphor becomes conventionalized (Bowdle \& Gentner, 2005) - therefore metaphor familiarity is also potentially important and, as has already been shown, activation magnitudes in relevant sensorimotor cortical areas decrease as familiarity increases in the case of action verbs used metaphorically (Desai et al., 2011). Because of these a priori hypotheses that metaphor familiarity and imageability might influence activation magnitude, we chose not to filter out their potential influences through the ANCOVA procedure. Similarly, we reasoned that variability in RTs might be related to aspects of metaphor processing and did not filter out RT in the ANCOVA. Instead, we used the ANCOVA option to test for voxels with significant correlations between activation magnitude and the semantic variables (familiarity and imageability), as well as RTs. These correlational analyses were carried out treating item as a random factor, as described above, but for the metaphorical sentences alone, and the resulting r-maps were cluster-corrected (voxel-wise $\mathrm{p}<.05$ ). These analyses were based on an 11-participant group for familiarity and sentence imageability since one participant did not complete the post-scan rating exercise; for RT, the entire 12-participant group was used.

We also tested $(n=12)$ for voxels showing significant correlations between activation magnitude and measures of individual propensity to engage in verbal information processing, or particular kinds of mental imagery characterized as object imagery or spatial imagery, based on the Object-Spatial Imagery and Verbal Questionnaire (OSIVQ, Blazhenkova \& Kozhevnikov, 2009). Object imagery refers to generation of highly depictive mental images that incorporate surface features of objects, whereas spatial imagery involves more schematic representations emphasizing spatial relationships between objects and object parts. We used the difference score between OSIVQ object imagery and spatial imagery ratings (OSIVQd) to index individual preference for object or spatial imagery (Lacey et al., 2011b), and the OSIVQ verbal score as a proxy for an individual's propensity for verbal 
coding.

\subsection{Resting-state functional connectivity (RSFC) analyses}

Using BrainVoyager, we followed the recommended MRI preprocessing methods for RSFC described by Weissenbacher et al. (2009) for seed-based correlation analysis: standard preprocessing included slice-timing correction, linear trend removal, retrospective 3D motion correction, spatial smoothing and spatial normalization to Talairach space. For structural scan averaging, five structural scans were acquired for each study participants (see 2.4 above). Each structural scan was Talairach-normalized, and the five normalized scans per participant were averaged, resulting in a structural data set for each participant with a high signal-to-noise ratio. White matter and ventricular tracings were accurately completed on the averaged structural data of each participant.

Selection of the seed for RSFC analyses was based on the activation data (see Results 3.3). Data correction for confounding effects originating from residual motion artifacts and physiologic signals (respiration/cardiac activity) was achieved through linear regression analysis: rigid-body realignment parameters from the retrospective motion correction of 3DPACE and mean white matter and lateral ventricle signals were used as regressors. The resulting residuals from each subject were combined in a multi-subject random-effects GLM. This yielded a t-map of RSFC with the seed, which was manually set at an uncorrected voxel-wise $\mathrm{p}<.0001$, and corrected for multiple comparisons (corrected $\mathrm{p}<$. $05)$ with the cluster correction approach. The cluster threshold of this group map was 5 voxels.

In addition, we examined whether the strength of RSFC with the seed correlated across participants with the OSIVQ verbal score and the OSIVQd score, reflecting individual preferences for, respectively, verbalizing and object vs. spatial imagery. We were particularly interested in whether the r-maps (voxel-wise $\mathrm{p}<.05$; cluster-corrected in the whole brain as in 2.7 above) identified foci overlapping with or adjacent to the RSFC t-maps 
described in the preceding paragraph, i.e. whether voxels exhibiting significant correlations with the OSIVQ parameters across participants coincided with or were near voxels demonstrating significant RSFC with the seed region in the entire group.

\subsection{Effective connectivity analysis}

We examined directional brain connectivity during metaphor processing using multivariate Granger causality analysis (GCA) on regions of interest (ROIs) selected based on the activation and RSFC analyses (see Results). A customized algorithm implemented in MATLAB (www.mathworks.com), described in detail in previous reports (Grant et al., 2014; Lacey et al., 2014; Sathian et al., 2013; Hampstead et al., 2015) was utilized to estimate the directional influence between ROIs (Deshpande et al., 2009; Deshpande \& Hu, 2012; Deshpande et al., 2012; Friston et al., 2003; Preusse et al., 2011; Roebroeck et al., 2005; Sathian et al., 2011; Stilla et al., 2007, 2008; Strenziok et al., 2010). GCA is based on the concept of temporal precedence across multiple time series (Granger, 1969). For example, given two time series $\mathrm{X}$ and $\mathrm{Y}$, if including the past value of $\mathrm{Y}$ can improve the prediction of the future value of $\mathrm{X}$, then a causal influence from Y to X can be inferred (Granger, 1969). Unlike dynamic causal modeling (DCM) which requires a pre-specified connectional architecture to investigate underlying directional connectivity between ROIs, the advantage of GCA is that it is a data-driven approach and thus can be applied in the absence of an $a$ priori model of underlying brain connectivity (Deshpande et al., 2009; Deshpande \& Hu, 2012; Deshpande et al., 2012; Friston et al., 2003; Preusse et al., 2011; Roebroeck et al., 2005; Sathian et al., 2011; Stilla et al., 2007, 2008; Strenziok et al., 2010). Recent reports of the validity of GCA in experimental applications (Abler et al., 2006) and simulations (Deshpande et al., 2010b; Wen et al., 2013) have shown its advantages and validity for estimating effective brain connectivity.

Mean time series of selected ROIs (see 3.3 for selection criteria) were extracted from $5 \mathrm{~mm}$ cubes centered at the peak activation maxima. Latent neural signals underlying these mean time series were obtained using blind hemodynamic deconvolution using a cubature Kalman 
filter, which has been shown to be the most comprehensive model to date for jointly estimating voxel-specific latent neural variables as well as hemodynamic response functions (HRFs) (Havlicek et al., 2011). Recent research has also shown that this parameterized blind deconvolution method does not over-fit and produces estimates that are highly correlated with those obtained from model-free, non-parametric methods (Sreenivasan et al., 2015). Hemodynamic deconvolution removes HRF variability across different participants and different regions of the brain (Handwerker et al., 2004), as well as the smoothing effect of the HRF. Specifically, the underlying model used for deconvolution is formulated in continuous time and was discretized here at a resolution of TR/10 as suggested by Havlicek et al. (2011). This leads to an increase in the effective temporal resolution of the latent neural signal used in GCA.

The resulting latent neural responses underlying the BOLD time series from the selected ROIs were input into a first-order, dynamic, multivariate, autoregressive model (dMVAR) (Grant et al., 2015; Hutcheson et al., 2015; Wheelock et al., 2014; Kapogiannis et al., 2014) to obtain measures of directional influence between multiple selected ROIs as a function of time (Grant et al., 2015; Hutcheson et al., 2015; Wheelock et al., 2014) such that only direct connections are considered (Deshpande et al., 2008, 2010a; Stilla et al., 2007). Because of our interest in causal influences arising from neural delays which are less than a TR (Deshpande et al., 2013), a first-order model was implemented. The time-varying coefficients of the dMVAR model are representative of effective connectivity between regions. Path weights at those times corresponding to specific conditions for each participant were obtained. The condition-specific path weights from all participants were then populated into two samples and a two sample t-test was performed to reveal paths having significantly higher $(\mathrm{p}<.05)$ effective connectivity in the condition of interest as compared to the control condition. The resulting significant paths between brain regions were displayed on a brain surface using a graphical interface visualization tool (Xia et al., 2013) known as BrainNet Viewer (www.nitrc.org/projects/bnv/). 


\section{RESULTS}

\subsection{Behavioral}

Accuracy on the valence task was higher for metaphorical sentences $(76.25 \% \pm 3.52 \%)$ $($ mean $\pm \mathrm{SE})$ than their control sentences $(71.33 \% \pm 2.47 \%)\left(\mathrm{t}_{11}=2.38, \mathrm{p}<.05\right)$. RTs were faster for metaphorical sentences $(1121 \mathrm{~ms} \pm 87 \mathrm{~ms})$ than metaphor control sentences $(1215 \mathrm{~ms}$ $\pm 91 \mathrm{~ms})\left(\mathrm{t}_{11}=-2.24, \mathrm{p}<.05\right)$, even though metaphorical sentences were longer than their control sentences $(1587 \mathrm{~ms}$ vs. $1481 \mathrm{~ms})$. RTs were uncorrelated with sentence duration for both metaphors $(\mathrm{r}=-.15, \mathrm{p}=.25)$ and metaphor controls $(\mathrm{r}=.1, \mathrm{p}=.5)$. RTs were negatively correlated with metaphor familiarity $(r=-.35, \mathrm{p}<.01)$, i.e. RTs became faster as familiarity increased, but were uncorrelated with sentence imageability for either metaphors $(r=-.14, p$ $=.3)$ or controls $(\mathrm{r}=-.1, \mathrm{p}=.5)$. There was no significant difference in accuracy on the valence task between the literal sentences $(77.17 \% \pm 5.2 \%)$ and their controls $(73.58 \% \pm$ $5.1 \%)\left(\mathrm{t}_{11}=1.3, \mathrm{p}=.21\right)$, but RTs were faster for literal sentences $(1229 \mathrm{~ms} \pm 119 \mathrm{~ms})$ than literal control sentences $(1382 \mathrm{~ms} \pm 145 \mathrm{~ms})\left(\mathrm{t}_{11}=-2.85, \mathrm{p}<.02\right)$.

Analysis of participants' post-scan ratings showed that the metaphor group (i.e. all metaphorical sentences plus their controls) was rated as less imageable than the literal group (4.06 vs $5.48, \mathrm{~F}_{1,10}=16.5, \mathrm{p}=.002$ ) but there was no significant difference in familiarity (5.56 vs $5.41, \mathrm{~F}_{1,10}=.9, \mathrm{p}=.4$ ). Compared to all the control sentences, the experimental sentences (i.e. all metaphorical and literal sentences) were rated more imageable (4.98 vs $\left.4.55, \mathrm{~F}_{1,10}=6.9, \mathrm{p}=.025\right)$ but less familiar (5.35 vs $\left.5.62, \mathrm{~F}_{1,10}=5.3, \mathrm{p}=.04\right)$. There was a significant group $x$ sentence type interaction for familiarity $\left(\mathrm{F}_{1,10}=12.4, \mathrm{p}=.006\right)$. Metaphorical sentences were rated significantly less familiar than their controls (5.16 vs $\left.5.96, \mathrm{t}_{10}=-3.7, \mathrm{p}=.004\right)$ while literal sentences were not (5.54 vs $\left.5.28, \mathrm{t}_{10}=1.6, \mathrm{p}=.1\right)$ - as was also the case in the pre-experiment norming exercise; the metaphorical and literal sentences themselves were not significantly different $\left(t_{10}=-1.4, p=.2\right)$. The group $x$ sentence type interaction was not significant for the post-scan imageability ratings $\left(\mathrm{F}_{1,10}=.5\right.$, $\mathrm{p}=.5)$. 


\subsection{Imaging}

\subsubsection{Localizer scans}

For the purpose of testing whether processing of body-part metaphors is grounded in somatic sensorimotor cortical activity, we focused on activity in the left hemisphere, since the motor conditions involved right-sided movement and the somatosensory conditions involved rightsided stimulation.

In the motor and somatosensory localizers, as would be expected on the basis of well-known somatotopic organization, there were body-part regions exhibiting high specificity. Balanced contrasts of each body part against the other two, relative to baseline, showed motor activation (corrected using the false discovery rate (FDR) method (Genovese et al., 2002), q $<.05)$ in the left precentral gyrus, which includes primary motor cortex (M1), for each body part. Using the same balanced contrasts, there was somatosensory activation in left primary somatosensory cortex (S1) (left postcentral gyrus [poCG]; cluster-corrected, voxel-wise $\mathrm{p}<$. 01). We used these body part-specific contrasts because of the established somatotopy in these regions, and did not consider motor or somatosensory responses outside these regions.

For the visual localizer, we used a balanced contrast of arms + legs $>$ objects to identify limb-selective activity and a separate contrast of faces $>$ objects to localize face-selective activity. The former contrast showed activation bilaterally in the posterior middle temporal gyrus (pMTG) extending to the middle occipital gyrus (MOG: cluster-corrected, voxel-wise $\mathrm{p}<.05$ ), comparable to previous reports of the EBA (Downing et al., 2001; Taylor et al., 2007; Myers \& Sowden, 2008; Weiner \& Grill-Spector, 2010, 2011, 2013); hereafter, this region is referred to as the EBA, for simplicity. However, we could not reliably localize the FFA (Kanwisher et al., 1997) or other body part-specific visual regions, such as the fusiform body area (FBA: e.g. Weiner \& Grill-Spector, 2013) since activity in these areas did not survive correction for multiple comparisons. 
These motor, somatosensory and visual localizers were defined based on thresholding the relevant activations to allow sufficient extents for hypothesis testing and, in the case of the somatic sensorimotor activations, to balance this need with that for separability of the body part-specific activations. The latter activations were constrained to the specific anatomic regions noted above. To guard against the possibility that the variations in thresholding between the different localizers favored one region over another, and to allow for the possibility of metaphor-specific activations near, but not within, the regions resulting from the localizers, e.g. according to the anterior-shift hypothesis (Chatterjee 2010; Chen et al., 2008), the analyses of language-related activations were conducted on a whole-brain basis (with correction for multiple comparisons in the whole brain) and the resulting loci were compared with the regions derived from the localizers. The variations in thresholding turned out to be of no consequence, as no language-related activations were found in or near the localizer-derived regions, with the exception of the left EBA (see below).

\subsubsection{Language tasks}

\subsubsection{Participant-based analyses}

These analyses were conducted in the hierarchical manner recommended by Nieuwenhuis et al. (2011). Considering activations evoked by metaphorical sentences relative to their controls and those evoked by literal sentences relative to their controls as the key activations of interest, there are three a priori possibilities concerning their relationship: the former could be greater, signifying body part metaphor-specific activation; the latter could be greater, signifying activation preferentially for literal use of body part words; or the two activations could be equal in magnitude. These possibilities were distinguished by testing for interaction effects as outlined below. Subsequent post-hoc contrasts were then applied to tease out specific effects.

\subsection{Metaphor-specific activation}

We began by testing for activity that was metaphor-specific, i.e. activity specific for bodypart metaphors relative to their controls, but not generalizing to literal use of body-part 
words. This was tested using the interaction contrast of (metaphor $>$ metaphor control) $>$ (literal $>$ literal control), cluster-corrected in the whole brain (voxel-wise $p<.05$, cluster threshold 57 voxels), which revealed bilateral activity in probable auditory cortex (superior temporal gyrus [STG]), extending inferiorly into the superior temporal sulcus (STS) and anteriorly into the inferior frontal gyrus (IFG). In the left hemisphere, this activity was more extensive and also extended posteriorly into the MTG, forming a contiguous belt (Figure 2a). Given the finding of metaphor-specific activity, the possibility of equal activation in metaphor and literal usages was not supported. The reverse whole brain interaction contrast of $($ literal $>$ control $)>($ metaphor $>$ control $)$ did not reveal any activity specific to literal usage of body part words.

Next, we examined post-hoc contrasts of metaphor $>$ control and literal $>$ control, within a mask of the metaphor-specific interaction activation. Since we tested two post-hoc contrasts, a Bonferroni correction was applied by setting the voxel-wise threshold to $\mathrm{p}<.025$ before cluster-correcting. The contrast of metaphor $>$ control showed that, relative to their controls, metaphorical sentences activated foci (cluster-corrected, cluster threshold 11 voxels) bilaterally in the STG and in the left posterior MTG (Figure 2b). The latter focus was located in the anterolateral part of the region identified on the visual localizer as the limb-selective EBA, and extending slightly laterally outside the visually responsive region at its anterior aspect, at the thresholds used. The contrast of literal > control showed that literal sentences did not evoke any significant activity relative to literal control sentences. In fact, literal control sentences evoked more activity than the literal sentences, particularly in bilateral STG, STS, and IFG, and more extensively in the left hemisphere than the right (cluster corrected, cluster threshold 25 voxels; data not shown) which explains why the reverse interaction contrast (see above) did not show any activity.

The whole-brain interaction analyses did not reveal metaphor-selective activity in any areas known to be visually face-selective. This might reflect a lack of power since there were only 20 items for each body part category. Arguing against this possibility, at the peak voxel of the 
left EBA metaphor-specific activation, there were significant results separately for armrelated metaphors $(\mathrm{t}=2.62, \mathrm{p}=.02)$, and leg-related metaphors $(\mathrm{t}=4.01, \mathrm{p}=.002)$, but not for face-related metaphors $(\mathrm{t}=-.03, \mathrm{p}=.98)$. Nor was there metaphor-selective activity in other cortical areas selectively responsive to visual images of body parts (Weiner \& GrillSpector, 2013). Additionally, we did not observe any activity in the motor or somatosensory ROIs in relation to either metaphorical or literal sentences.

\subsection{Limb metaphor-specific activation in the EBA}

The EBA was localized in the present study from the contrast of limbs vs. objects based on its well-known visual selectivity for images of limbs (Downing et al., 2001; Taylor et al., 2007; Myers \& Sowden, 2008; Orlov et al., 2010; Weiner \& Grill-Spector 2010, 2011, 2013). Therefore, we tested whether the metaphor-selective activity in the left EBA was limbspecific by carrying out the contrast (limb metaphor $>$ limb control) $>$ (face metaphor $>$ face control) within a mask of the activations resulting from the interaction contrast (which used the entire sets of sentences, see above). This resulted in virtually the same L EBA focus as the post-hoc test for all metaphors vs. controls (see above), but no activity was seen outside the EBA, i.e. the STG activity was not specific to limb metaphors (Figure 2). We further calculated effect sizes and 95\% confidence intervals (95\% CI) (Lacey et al., 2011a, 2012) for left EBA responses in order to describe these more fully while also obviating a particular concern for small samples such as ours, which is their association with wide confidence intervals that can result in spuriously large effect sizes (Gardner \& Altman, 1986). We extracted the baseline-referenced z-transformed beta weights from the left EBA in the limb metaphor and corresponding control conditions for each participant. Using these, Cohen's d was computed to be .46 (95\% CI: $.27-.65)$; thus there was a medium size effect with narrow confidence intervals that were clearly distinct from zero. Given that the metaphor-selective EBA focus was displaced anterolaterally, relative to the center of gravity of the visuallyselective EBA regions identified from the visual localizer (Figure 3), we wanted to confirm that this metaphor-related focus also reflected the visual preference for limb images relative to objects that characterizes the EBA as a whole. To this end, we calculated the effect size for 
visual images of limbs vs. objects at this metaphor-selective focus. Cohen's d was .92 (95\%

CI: $.53-1.04)$; this large effect size, again with narrow confidence intervals that were clearly distinct from zero, verified that the metaphor focus was also limb-selective (relative to objects) for visual stimuli. Note that these effect size computations at the metaphor-selective focus were not intended as independent analyses; they were performed merely to allow further characterization of the responses.

\subsection{Correlations with OSIVQ scores}

There was an extensive set of areas where activation magnitudes during processing of limbrelated metaphors correlated with OSIVQ verbal scores $(\mathrm{p}<.05, \mathrm{r}=.58$, whole brain clustercorrected, threshold 76 voxels; Supplementary Table 1) but none of these were in the left EBA or cerebral areas potentially associated with language processing; however, there were a number of bilateral cerebellar foci. The right cerebellum especially is functionally and structurally connected to language areas of the cerebral cortex (Mariën et al., 2014), though whether it has a special role in metaphor processing is not clear. Correlations of activation magnitudes during processing of limb-related metaphors with OSIVQd scores were negative $(\mathrm{p}<.05, \mathrm{r}=.58$, whole brain cluster-corrected, threshold 67 voxels; data not shown), meaning that activation magnitudes decreased with increasing preference for object compared to spatial imagery, but the left EBA and language-related areas were similarly absent from this set of areas.

\subsubsection{Item-based analyses}

The above analyses generalize to untested participants, since participant was treated as a random factor. To test for generalization of our results across items, we next ran an ANCOVA treating item as a random factor, with the item-wise acoustic variables (mean pitch, mean intensity and sentence duration) treated as nuisance covariates. Since the participant-based analyses did not reveal activity in any visually face-selective areas, and the EBA is visually limb-selective (see above), we restricted the ANCOVA to the limb metaphors (along with their respective controls, see Methods). Since we were interested in areas showing metaphor- 
specific activity, this analysis was conducted within a mask comprising the bilateral STG and left EBA metaphor-selective regions yielded by the post-hoc metaphor $>$ metaphor control contrast from the participant-based analyses. On this ANCOVA, only the left EBA focus was significantly active for limb metaphors relative to their controls (cluster-corrected, voxelwise $\mathrm{p}<.05$, threshold 10 voxels) (Figure 2). (Note that, in order to avoid a possible violation of smoothness associated with small ROIs, we thresholded the mask used for the ANCOVA at $\mathrm{p}<.05$ rather than the Bonferroni-corrected $\mathrm{p}<.025$; however, $70 \%$ (38 voxels) of the resulting left EBA focus fell within the Bonferroni-corrected activation reported above, see Figure 3). The bilateral STG activity found on the participant-based analyses did not appear in the ANCOVA result and was therefore likely related to acoustic stimulus properties (which were factored out in the ANCOVA), rather than to metaphor processing per se. The left EBA focus was therefore the only focus active in all analyses, i.e. it exhibited metaphor-selective activity for limb metaphors that generalized across both participants and items.

We also conducted correlational analyses, again treating item as a random factor, within the same mask as above and on limb metaphors only. There were no regions with significant correlations between activation magnitudes and metaphor familiarity or imageability. There was a significant, negative correlation with RTs in the right STG (cluster-corrected, voxelwise $\mathrm{p}<.05$, threshold 10 voxels), i.e. increased activation magnitude was associated with faster RTs.

\subsection{RSFC analyses}

We used the resting-state fMRI data to examine the RSFC of the limb metaphor-specific focus in the left EBA. Thus, we used a seed ROI, which was a $5 \mathrm{~mm}$ cube (centered at -45 , $-55,13)$ in the left EBA, derived from the post-hoc metaphor > control contrast that followed the interaction analysis (see 3.2.2.1 above). This revealed an extensive network of areas (Figure 4 and Supplementary Table 2) including, most interestingly, language-related areas: foci in the pars triangularis (PT) of Broca's area in the left inferior frontal gyrus (IFG) 
(Keller et al., 2009); the left angular gyrus (AG), a part of inferior parietal cortex suggested to be a key player in metaphor processing (Ramachandran \& Hubbard, 2001; Benedek et al., 2014; Obert et al., 2014); and left anterior temporal foci in the STG and ITS, potentially involved in semantic processing (Lambon Ralph et al., 2010a,b, 2012). The left supramarginal gyrus (SMG), another inferior parietal focus, was also present in the network; this area has been implicated in both language (Deschamps et al., 2014) and somatosensory processing (Van de Winckel et al., 2005). Interestingly, there were also resting-state connections to other visual cortical regions at loci known to respond to images of body parts or faces: a body-part responsive focus in the left lateral occipito-temporal sulcus (OTS) (Weiner \& Grill-Spector, 2013), and bilateral MOG foci corresponding to the occipital face area (OFA) (Myers \& Sowden, 2008). Further, there were connections between the metaphorspecific EBA locus and bilateral visual regions in the calcarine sulcus, parieto-occipital fissure (POF), and precuneus, perhaps reflecting visual imagery (e.g. Fletcher et al., 1995), and bilateral somatic sensorimotor regions in precentral and postcentral gyri. Additionally, the network involved other foci in prefrontal and superior temporal cortex, and various subcortical structures including the amygdala and a number of bilateral cerebellar foci. Apart from a small area in the left STG extending into the STS, however, none of these areas were activated on the post-hoc metaphor $>$ control contrast described in 3.2.2.1.

We also examined the RSFC data for correlations across individuals between the strength of RSFC to the EBA metaphor-specific seed and OSIVQ parameters. For the OSIVQ verbal score, the resulting $\mathrm{r}$-map (thresholded at $\mathrm{p}=.05, \mathrm{r}=.58$; whole brain cluster-corrected, threshold 49 voxels) demonstrated correlated voxels in Broca's area (in both PT and pars opercularis [PO]), the left AG, and the left anterior temporal lobe in the STS, as well as a number of other areas (Figure 4 and Supplementary Table 3). Many of these voxels coincided with or were near voxels identified as exhibiting RSFC across the entire participant group with the EBA seed. These included left hemisphere foci in the IFG including the one in the PT of Broca's area, AG, posterior STG, the anterior temporal lobe in the STS, the precuneus 
and anterior calcarine sulcus; right hemisphere foci in the superior parietal gyrus (SPG), postcentral sulcus (poCS), precentral gyrus (preCG) and STS; and bilateral foci in the POF.

For the OSIVQd score, the resulting r-map (thresholded at $\mathrm{p}=.05, \mathrm{r}=.58$; cluster-corrected, threshold 49 voxels) only showed isolated areas at (right MTG and subcortical areas) or near (right AG and inferior central midbrain) voxels that had significant RSFC with the EBA seed.

\subsection{Effective connectivity}

Next, we examined effective connectivity during limb metaphor processing. To do so, we selected a set of ROIs including the limb metaphor-specific EBA focus (the seed for the primary RSFC analysis). Since there were no other areas whose activity was specific to processing of limb metaphors, the other ROIs for effective connectivity analysis were chosen from the resting-state data. We included all foci showing correlations between the strength of RSFC with the left EBA seed and the OSIVQ verbal score that also featured in, or were adjacent to, foci exhibiting significant RSFC with the EBA seed. In cases where two foci from the same region satisfied these criteria, the focus with the larger $r$ value was chosen. 13 ROIs emerged from this selection process (bolded in Supplementary Table 3). We also included additional ROIs: since our major focus was to define effective connectivity of the EBA with language-related and sensorimotor areas, this guided the remainder of the selection process. One of these ROIs, the left PO (part of Broca's area), showed a correlation between the strength of RSFC with the left EBA seed and the OSIVQ verbal score (bolded in Supplementary Table 2). The other 8 (also bolded in Supplementary Table 2) were selected from the regions showing significant RSFC with the EBA seed and included foci in: the left PT (part of Broca's area, distinct from the focus in Supplementary Table 3); the left anterior temporal lobe in the STG and ITS; the left preCG (M1); the left poCG (S1); the left posterior insula (pIns), another site of somatosensory processing corresponding to the medial part of parietal opercular fields that exhibit responsiveness to tactile stimulation of the leg (Eickhoff et al., 2007); the right MOG comprising part of the right EBA from the visual localizer; the 
left supramarginal gyrus (SMG); and the left amygdala in view of the valence task that participants performed during fMRI scanning while listening to metaphors.

Effective connectivity between these 23 ROIs was estimated as detailed in the Methods section. Path weights corresponding to the limb metaphor and limb control conditions for participant were obtained and populated into two samples, one for each of these conditions. Since we were interested in path weights that were greater in the limb metaphor compared to the limb control condition, a one-tailed, two sample t-test was performed to reveal the significant paths $(\mathrm{p}<.05)$ on this comparison. Inspection of the directional patterns in the resulting network (Figure 5) revealed that metaphor-related activity in the left EBA focus was driven by language-related areas: one of the PT foci of Broca's area and a left anterior temporal focus in the STG. The latter focus was a major driver in the network, also providing input to other language-associated areas: the left PO of Broca's area and another left anterior temporal lobe focus in the ITS; somatosensory areas (right PCS and left pIns); a visual area (right POF); and the right STS, a multisensory region. The PT focus driving the EBA also provided input to the other, more anterior, PT focus and the left POF. The SPG focus projected to an IFG focus superior to Broca's area while the left precuneus projected to the right POF, and both the right SPG and the left precuneus foci provided input to the left anterior STG driving hub. Note that, of the 15 ROIs contributing to the effective connectivity network, 10 were from the set where RSFC was significantly correlated across participants with the OSIVQ verbal scores and also overlapped with RSFC regions that connected with the EBA seed.

\section{DISCUSSION}

In this study we tested the prediction of conceptual metaphor theory that metaphor comprehension involves activation of sensorimotor cortices involved in processing the domain from which the metaphor is drawn. The main findings are that processing limbrelated body-part metaphors recruits activity in the functionally-localized left EBA, a visual limb-selective region (Downing et al., 2001; Taylor et al., 2007; Myers \& Sowden, 2008; 
Orlov et al., 2010; Weiner \& Grill-Spector 2010, 2011, 2013) and that the left EBA has both resting-state and effective connectivity with language areas. Although the sample size in the present study is relatively small, it is comparable to that in Aziz-Zadeh et al. (2006) and Chen et al. (2008) and larger than that used in our earlier study of texture metaphors (Lacey et al., 2012). Further, the main finding of limb metaphor-evoked activity in the left EBA is robust enough to appear in both item- and participant-based analyses of activation; and we provide converging evidence from both resting-state and effective connectivity analyses. These findings extend and reinforce prior observations that metaphors drawn from particular domains elicit activity in or near the related domain-specific sensorimotor cortical regions (Aziz-Zadeh et al., 2006; Boulenger et al., 2009; Desai et al., 2011, 2013; Lacey et al., 2012).

\subsection{Body-part metaphor processing in the EBA}

The participant-based analyses showed that sentences containing body-part metaphors elicited activations, relative to control sentences matched for meaning, in the left EBA and bilateral STG (probable auditory cortex), but no preferential activity was found anywhere for the body literal sentences. Critically, the significant interaction effect of sentence type (metaphorical/literal vs. experimental/control) confirmed that the differential effect for metaphorical compared to literal usage of body-part words was not simply due to statistical threshold effects (Nieuwenhuis et al., 2011). Given the known visual selectivity of the EBA for images of limbs (Weiner \& Grill-Spector, 2011, 2013), we also verified that the left EBA metaphor-selective activation focus was selective for limb metaphors relative to face metaphors, again using an interaction contrast involving the respective controls. Effect sizes for both limb metaphors (relative to their controls) and visual images of limbs (relative to images of objects) displayed narrow confidence intervals that did not overlap zero, arguing against the possibility that the metaphor-selective effect was an artifact of a small sample size (Gardner \& Altman, 1986). Item-based analysis of the limb metaphors relative to their controls also showed activation in the left EBA after accounting for the nuisance variables of mean pitch and intensity and sentence duration. Thus, EBA metaphor-specific activity was limb-selective and is generalizable to untested participants as well as untested limb 
metaphors. STG activity, however, was not limb-selective and was not found on the itembased analyses. Given its location in probable auditory cortex, this activity may reflect the significantly longer duration of metaphorical compared to control sentences (Table 1b). The reason for the negative correlation of right STG activation magnitude with RT is uncertain, but may reflect more efficient processing associated with faster responses.

Along the lines of the proposed posterior-to-anterior shift in neural processing as one proceeds from concrete to abstract concepts or usages (Chatterjee, 2010; Chen et al., 2008), the left EBA metaphor-selective activation occupied the anterolateral part of the EBA activation produced by visual processing of body parts during the localizer task, extending slightly lateral to the visual ROI at its anterior aspect. The visually responsive EBA extended from left pSTS inferiorly through pMTG to pITS and posteriorly to left MOG, consistent with previous studies (Downing et al., 2001; Taylor et al., 2007; Myers \& Sowden, 2008; Weiner \& Grill-Spector, 2010, 2011, 2013); the metaphor responsive EBA focus was in the left pMTG, i.e. the anterior part of the visual EBA. Although the details of the relationship between the metaphor-specific EBA focus and the visually responsive EBA are dependent on the precise thresholds used, the anterolateral displacement of the center of gravity of the metaphor response relative to that of the visual response is threshold-independent. Thus, relative to the focus of visual responsiveness in the EBA, metaphor-evoked activity was displaced anterolaterally, while still retaining visual responsiveness to limb-images. We infer that the neuronal pools mediating conceptual and perceptual processing are not identical but only partially shared.

The left lateralization of the EBA metaphor-specific focus reflects the general finding that processing non-literal language is predominantly left-lateralized. The right hemisphere does not seem to be preferentially involved in processing metaphors (Bohrn et al., 2012; Rapp et al., 2012; Vartanian, 2012; Yang, 2014) except when they are novel (Bohrn et al., 2012; Yang, 2014: see also Kasparian (2013) for a critical review of a special role for the right hemisphere). However, other accounts argue that as metaphors become conventionalized, i.e. 
more familiar, there is a reduction of the neural load in bilateral semantic networks, rather than a hemispheric shift in processing (Cardillo et al., 2012). In previous studies, areas involved in motor control and the perception of biological motion showed an inverse relationship between activation magnitude and the familiarity of both metaphorical and literal expressions using action verbs (Desai et al., 2011), in keeping with weakening of the simulations postulated to underlie metaphor comprehension as the metaphors become more familiar (Bowdle \& Gentner, 2005). Although we did not find a statistically significant effect of metaphor familiarity, our metaphor set contained mostly familiar metaphors and was not explicitly designed to test for such a relationship. Similarly, we found no relationship between activation magnitude and sentence imageability, arguing against a simplistic visual imagery explanation for the EBA activations. Note however, that grounded cognition accounts argue for the importance of perceptual simulations, of which visual imagery is a prime example (Barsalou, 2008); thus, a visual imagery explanation would not necessarily conflict with conceptual metaphor theory. A caveat to our failure to find significant correlations between activation magnitude and either familiarity or imageability is the relatively small sample size; thus, these relationships cannot be completely ruled out by the present study.

Finally, we need to deal with two potential confounds. Firstly, since the metaphorical sentences were rated as less interpretable than their control sentences, it might be argued that the present results reflect this increased complexity rather than their metaphoric nature per se. However, this is unlikely given that RTs were significantly faster for metaphors than their controls. In addition, of the areas shown by the metaphor > control contrast, the STG activation was clearly related to acoustic, rather than linguistic, properties of the stimuli. Secondly, we chose controls that matched the metaphors for meaning. We attempted to minimize syntactic differences between metaphorical and control sentences but acknowledge that such differences were not fully eliminated. However, we did not observe differential activation in the language network for metaphors relative to their controls, as might be 
expected if syntactic differences were salient, and is it not obvious why any residual syntactic mismatches should evoke EBA activity.

\subsection{Body-part metaphor processing in motor and somatosensory cortex}

Perhaps surprisingly, there was no metaphor-specific activity in classical motor or somatosensory areas. For motor areas, at least, this might suggest that, while they might be engaged by action words that imply a related body part (Aziz-Zadeh et al., 2006; Boulenger et al., 2009), they are either not engaged by explicit mention of body parts regardless of implied action, or there was insufficient implied action in the sentences used in the current study. We should note, too, that previous studies have not consistently shown activity in motor cortex for figurative language: neither action metaphors (Raposo et al., 2009) nor motion idioms (Chen et al., 2008) activated motor areas. The EBA is not a classical motor area; however, it is noteworthy that it does seem to have some association with motor behavior, although its precise role remains uncertain (Downing \& Peelen, 2011). The null effect in classical somatic sensorimotor areas does not fully exclude a role for these areas in processing body part metaphors, which could potentially be revealed with a larger sample size or alternative analyses. However, if this null effect is true, it may reflect a subtle distinction between 'grounded' and 'embodied' cognition, although these terms are often used interchangeably. While 'embodied cognition' conceptualizes metaphor comprehension

as being specifically related to bodily experience (thus implicating motor and somatosensory cortex), 'grounded cognition' posits that metaphors could be grounded in many ways that connect to the external world (e.g., here, visual perception of others' bodies) which are not limited to bodily experience per se (Borghi et al., 2013). Thus, the current results favor grounded, rather than embodied, metaphor processing. Either way, given the EBA response, the lack of metaphor responses in motor or somatosensory cortices is not in itself an argument against conceptual metaphor theory.

\subsection{Face metaphors}


We did not see metaphor-related activity in any areas known to process visual face stimuli. One explanation may be that OFA, and to some extent FFA, have a faciotopic organization with separable cortical areas selectively processing eyes, ears nose, mouth and so on (Henriksson et al., 2015) and that we simply lacked enough feature-specific metaphors to activate these. For example, there were only two 'eye' and three 'nose' metaphors in our set. This assumes featural face processing but even if we assume configural processing, there were only six 'head' and four 'face' metaphors in our set. Furthermore, body-part representations in EBA are at least partly semantically organized (Bracci, Caramazza \& Peelen, 2015) which may facilitate processing of limb metaphors. It is not known whether such semantic organization is present in OFA or FFA but, if it were in fact absent, this might be a contributing factor to the absence of face metaphor activity in these areas. It is also possible that, with only 20 metaphors, there was a general lack of power to detect facemetaphor activity, but this seems unlikely given that we were able to obtain arm- and legspecific results with the same number of metaphors at the peak voxel of the left EBA, an area that also has part-specific topology (Orlov et al., 2010), if not actual somatotopy. Face metaphors were rated as slightly, but not significantly, more familiar than limb metaphors (5.4 vs 4.8); since activation magnitudes decrease with increasing familiarity this might have reduced the overall activity for face metaphors, although it seems unlikely that this small disparity could have eliminated it altogether.

\subsection{Body-part words in literal contexts}

Unlike their metaphorical use, the use of body parts in literal contexts did not evoke activity in body-related cortex or, indeed, anywhere else. This may reflect deeper semantic processing of metaphors compared to the literal sentences, which refer to body parts in highly overlearned contexts. Indeed, although the scanned participants rated the metaphorical and literal sentences as equally familiar, they did rate the metaphorical, but not the literal, sentences as significantly less familiar than their respective controls. Increased familiarity of body-part words in everyday, literal usage might render extensive simulation and associated recruitment of sensorimotor cortex unnecessary, mere lexical access being sufficient. 
Supporting this possibility, a patient with a left occipitotemporal lesion that likely included the EBA was severely impaired at selecting the correct figurative body-part term referring to part of an inanimate object (e.g., the teeth of a comb or the arm of a chair), but had substantially better comprehension of body-part terms when used in familiar (overlearned) literal contexts (e.g., pointing to named body parts and understanding their locations and functions) (Kemmerer \& Tranel, 2008). In the present study, the control sentences that were paired with body-part literal sentences activated language-related areas (around STG, STS, and IFG) more than the latter sentences did, perhaps because the former were more engaging. The body-part literal sentences naturally referred to a very limited set of items, whereas the words that replaced body parts in the literal control sentences came from a variety of domains (e.g., shield, river, apple etc.) - this may simply have made the control sentences more interesting.

\subsection{Language-related network connectivity of the left EBA}

Analysis of RSFC showed that, apart from the left STG (probable auditory cortex), the left EBA was connected with an extensive network that was not revealed by the activation analyses (at least, at the statistical thresholds used). This network included language-related foci in Broca's area, the left anterior temporal lobe and the left inferior parietal cortex. Broca's area, of course, is a classical language area (Keller et al., 2009) which a recent metaanalysis shows to be preferentially active during metaphor processing relative to various control conditions (Bohrn et al., 2012). The left IFG more broadly may play a role in figurative language, being active during processing of both metaphors (Kasparian, 2013) and idioms (Yang \& Shu, 2015). The anterior temporal lobes are involved in concept formation and semantic memory (Lambon Ralph et al., 2010a,b, 2012) and are also implicated in metaphor processing (Bohrn et al., 2012). The presence of left anterior temporal foci in the EBA RSFC network may reflect the mapping between concepts necessary for metaphor formation and comprehension. The left AG is another potential language-related area in the RSFC network which is reported to be involved in metaphor processing (Ramachandran \& Hubbard, 2001; Benedek et al., 2014; Obert et al., 2014). The left, but not right, AG has 
recently been reported to be involved in semantic integration, i.e. deriving the semantic representation of 'black leather bag' from the component concepts of 'black', 'leather', and 'bag' (Price et al., 2016). Thus a potential role for the left AG in metaphor processing is the semantic integration of concepts from different domains to form metaphors. The left EBA metaphor-specific focus also exhibited resting-state connectivity with visual cortical regions selective for images of body parts or faces, such as the left lateral OTS body-part responsive focus (Weiner \& Grill-Spector, 2013), and bilateral OFA (Myers \& Sowden, 2008), and bilateral somatic sensorimotor regions in precentral and postcentral gyri. These connections with visual and somatic sensorimotor regions may reflect a network supporting various bodyrelated functions, such as extended processing of body or identity information, as well as grounding of body concepts in metaphors. Moreover, the EBA RSFC network also included other visual regions bilaterally in the calcarine sulcus, POF, and precuneus, perhaps reflecting visual imagery (e.g. Fletcher et al., 1995). Underscoring the potential relevance of the connectivity of the EBA metaphor-specific seed to language-related areas, many of these connections demonstrated connectivity strengths that were correlated with individual OSIVQ verbal scores. Similar correlations also characterized cortical foci bilaterally in the POF and precuneus, and multiple foci in the right SPG.

In accordance with a grounded account of metaphor processing, the metaphor-specific left EBA focus was driven by two language-related foci, one in the PT of Broca's area and the other in the anterior temporal lobe (in the STG). The likely anatomical basis for connectivity relationships between Broca's area and the left EBA is the inferior fronto-occipital fascicle (IFOF: Sarubbo et al., 2013, 2015). The superficial layer of the IFOF connects extrastriate cortex to Broca's area, and is thought to subserve semantic processing (Sarubbo et al., 2013). The effective connectivity analysis also revealed projections between the anterior STG focus and Broca's area: this connectivity is probably via the extreme capsule (Frey et al., 2008). The anterior STG focus emerged as the principal driving hub in the effective connectivity network, providing inputs to multiple sensory regions in addition to the EBA and Broca's area. 


\subsection{Conclusions}

The present study shows that the left EBA, a visually limb-selective region, is activated during comprehension of limb-related metaphors, is connected with language-related regions in the resting state as well as during metaphor processing, and shows correlation of the strength of resting-state connectivity with language areas with individual verbal preference scores. The use of localizers and converging evidence from item- and participant-based activation analyses, RSFC analyses and effective connectivity analysis provides robust evidence for conceptual metaphor theory. The lesion study of Kemmerer and Tranel (2008) referred to above, suggests that the EBA is necessary for processing body-part terms metaphorically. Further application of the neuropsychological approach as well as the use of transcranial magnetic stimulation over the EBA will be helpful to elucidate the functional role of the EBA in metaphor processing, just as these approaches have helped delineate its functional role in perception and motor behavior (reviewed by Downing \& Peelen, 2015). Connectivity studies can also be expected to clarify how the EBA interacts with other areas, especially language areas, in the comprehension and production of body part metaphors.

\section{Acknowledgments}

This work was funded by NSF grant BCS 1125756 to KS. Support to KS from the Veterans Administration is also acknowledged. We are grateful to Lawrence Barsalou for his valuable insights, Stefania Bracci for kindly providing visual stimuli, and Lynne Nygaard for guidance on, and use of her laboratory and equipment, for acoustic analyses. 


\section{REFERENCES}

Abler, B., Roebroeck, A., Goebel, R., Höse, A., Schönfeldt-Lecuona, C., Hole, G., \& Walter, H. (2006). Investigating directed influences between activated brain areas in a motor response task using fMRI. Magnetic Resonance Imaging, 24:181-185.

Audacity Team (2012) Audacity v2.0.1 [Computer program]. Retrieved from http:// audacity.sourceforge.net/ Audacity ${ }^{\circledR}$ software is copyright (C) 1999-2014 Audacity Team.

Aziz-Zadeh, L., Wilson, S.M., Rizzolatti, G. \& Iacoboni, M. (2006). Congruent embodied representations for visually presented actions and linguistics phrases describing actions. Current Biology, 16:1818-1823.

Barsalou, L.W. (2008). Grounded cognition. Annual Review of Psychology, 59:617-645.

Bedny, M., Aguirre, G.K. \& Thompson-Schill, S.L. (2007). Item analysis in functional magnetic resonance imaging. NeuroImage 35:1093-1102.

Benedek, M., Beaty, R., Jauk, E., Koschutnig, K., Fink, A. et al. (2014). Creating metaphors: the neural basis of figurative language production. NeuroImage, 90:99-106.

Blazhenkova, O. \& Kozhevnikov, M. (2009). The new object-spatial-verbal cognitive style model: Theory and measurement. Applied Cognitive Psychology, 23: 638-663.

Boersma, P. \& Weenink, D. (2008). PRAAT: doing phonetics by computer. (v5.0.41) [computer program] Retrieved 23 November 2008 from http://www.praat.org/

Bohrn, I.C., Altmann, U. \& Jacobs, A.M. (2012). Looking at the brains behind figurative language - a quantitative meta-analysis of neuroimaging studies on metaphor, idiom, and irony processing. Neuropsychologia, 50:2669-2683. 
Borghi, A.M., Scorolli, C., Caligiore, D., Baldasarre, G. \& Tummolini, L. (2013). The embodied mind extended: using words as social tools. Frontiers in Psychology, 4:214 doi: 10.3389/fpsyg.2013.00214

Boroditsky, L. (2000). Metaphoric structuring: understanding time through spatial metaphors. Cognition, 75: 1-28.

Boulenger, V., Hauk, O. \& Pulvermüller, F. (2009). Grasping ideas with the motor system: semantic somatotopy in idiom comprehension. Cerebral Cortex, 19:1905-1914.

Bowdle, B.F. \& Gentner, D. (2005). The career of metaphor. Psychological Review, 112:193-216.

Bracci, S., Caramazza, A. \& Peelen, M.V. (2015). Representational similarity of body parts in human occipitotemporal cortex. Journal of Neuroscience, 35:12977-12985.

Bracci, S., Cavina-Pratesi, C., Ietswaart, M., Caramazza, A. \& Peelen, M.V. (2012). Closely overlapping responses to tools and hands in left lateral occipitotemporal cortex. Journal of Neurophysiology, 107:1443-1456.

Bracci, S., Ietswaart, M., Peelen, M.V. \& Cavina-Pratesi, C. (2010). Dissociable neural responses to hands and non-hand body parts in human left extrastriate visual cortex. Journal of Neurophysiology, 103:3389-3397.

Burton, A.M., White, D. \& McNeill, A. (2010). The Glasgow Face Matching Test. Behavioral Research Methods, 42:286-291. 
Cardillo, E.R., Watson, C.E., Schmidt, G.L., Kranjec, A. \& Chatterjee, A. (2012). From novel to familiar: tuning the brain for metaphors. NeuroImage, 59:3212-3221.

Casasanto, D. \& Boroditsky, L. (2008). Time in the mind: using space to think about time. Cognition, 106: 579-593.

Chatterjee, A. (2010). Disembodying cognition. Language \& Cognition, 2:79-116

Chen, E., Widick, P. \& Chatterjee, A. (2008). Functional-anatomical organization of predicate metaphor processing. Brain \& Language, 107:194-202.

Clark, H. (1973). The language-as-a-fixed-effect fallacy: critique of language statistics in psychological research. Journal of Verbal Learning \& Verbal Behavior, 12:335-359.

Coltheart, M. (1981). The MRC Psycholinguistic Database. Quarterly Journal of Experimental Psychology, 33A:497-505.

Desai, R.H., Binder, J.R., Conant, L.L., Mano, Q.R. \& Seidenberg, M.S. (2011). The neural career of sensory-motor metaphors. Journal of Cognitive Neuroscience, 23:2376-2386.

Desai, R.H., Conant, L.L., Binder, J.R., Park, H. \& Seidenberg, M.S. (2013). A piece of the action: modulation of sensory-motor regions by action idioms and metaphors. NeuroImage, 83:862-869.

Deschamps, I., Baum, S.R. \& Gracco, V.L. (2014). On the role of the supramarginal gyrus in phonological processing and verbal working memory: evidence from rTMS studies. Neuropsychologia, 53:39-46. 
Deshpande, G. \& Hu, X. (2012). Investigating effective brain connectivity from fMRI data: past findings and current issues with reference to Granger causality analysis. Brain Connectivity, 2:235-245.

Deshpande, G., Hu, X., Stilla, R., \& Sathian, K. (2008). Effective connectivity during haptic perception: A study using Granger causality analysis of functional magnetic resonance imaging data. Neuroimage, 40:1807-1814.

Deshpande, G., LaConte, S., James, G. A., Peltier, S., \& Hu, X. (2009). Multivariate Granger causality analysis of fMRI data. Human Brain Mapping, 30:1361-1373.

Deshpande, G., Sathian, K., \& Hu, X. (2010a). Assessing and compensating for zero-lag correlation effects in time-lagged Granger causality analysis of FMRI. IEEE Transactions in Biomedical Engineering, 57:1446-1456.

Deshpande, G., Sathian, K., \& Hu, X. (2010b). Effect of hemodynamic variability on Granger causality analysis of fMRI. Neuroimage, 52:884-896.

Deshpande, G., Sathian, K., Hu, X., \& Buckhalt, J. A. (2012). A rigorous approach for testing the constructionist hypotheses of brain function. Behavioral Brain Science, 35:148-149.

Deshpande, G., Libero, L. E., Sreenivasan, K. R., Deshpande, H. D., \& Kana, R. K. (2013). Identification of neural connectivity signatures of autism using machine learning. Frontiers in Human Neuroscience, 7:670.

Donoghue, D. (2014). Metaphor. Cambridge, MA: Harvard University Press.

Downing, P.E., Jiang, Y., Shuman, M. \& Kanwisher, N. (2001). A cortical area selective for visual processing of the human body. Science, 293:2470-2473. 
Downing, P.E. \& Peelen, M.V. (2011), The role of occipitotemporal body-selective regions in person perception. Cognitive Neuroscience, 2:186-203.

Downing, P.E. \& Peelen, M.V. (2015). Body selectivity in occipitotemporal cortex: causal evidence. Neuropsychologia, in press. doi: 10.1016/j.neuropsychologia.2015.05.033

Duvernoy, H.M. (1999). The Human Brain. Surface, Blood Supply and Three-dimensional Sectional Anatomy. New York: Springer.

Eickhoff, S.B., Grefkes, C., Zilles, K. \& Fink, G.R. (2007). The somatotopic organization of cytoarchitectonic areas on the human parietal operculum. Cerebral Cortex, 17:1800-1811.

Eviatar, Z. \& Just, M.A. (2006). Brain correlates of discourse processing: an fMRI investigation of irony and conventional metaphor comprehension. Neuropsychologia, 44:2348-2359.

Fletcher, P.C., Frith, C.D., Baker, S.C., Shallice, T., Frackowiak, R.S.J. \& Dolan, R.J. (1995). The mind's eye - precuneus activation in memory-related imagery. NeuroImage, 2:195-200.

Fodor, J. (1975). The Language of Thought. Cambridge, MA: Harvard University Press.

Forman, S.D., Cohen, J.D., Fitzgerald, M., Eddy, W.F., Mintun, M.A. et al. (1995). Improved assessment of significant activation in functional magnetic resonance imaging (fMRI): use of a cluster-size threshold. Magnetic Resonance in Medicine, 33:636-647.

Frey, S., Campbell, J.S.W., Pike, G.B. \& Petrides, M. (2008). Dissociating the human language pathways with high angular resolution diffusion fiber tractography. Journal of Neuroscience, 28:11435-11444. 
Friston, K. J., Harrison, L., \& Penny, W. (2003). Dynamic causal modelling. Neuroimage, 19:1273-1302.

Gardner, M.J. \& Altman, D.G. (1986). Confidence intervals rather than P values: estimation rather than hypothesis testing. British Medical Journal, 292:749-750.

Genovese, C.R., Lazar, N.A. \& Nichols, T. (2002). Thresholding of statistical maps in functional neuroimaging using the false discovery rate. NeuroImage, 15:879-878.

Gilhooly, J. \& Logie, R.H. (1980). Age-of-acquisition, imagery, concreteness, familiarity, and ambiguity measures for 1,944 words. Behavioral Research Methods \& Instruments, $12: 395-427$.

Granger, C. W. (1969). Investigating causal relations by econometric models and cross spectral methods. Econometrica, 37:424-438.

Grant, M. M., White, D., Hadley, J., Hutcheson, N., Shelton, R., Sreenivasan, K., \& Deshpande, G. (2014). Early life trauma and directional brain connectivity within major depression. Human Brain Mapping, 35:4815-4826.

Grant, M. M., Wood, K., Sreenivasan, K., Wheelock, M., \& White, D. (2015). Influence of Early Life Stress on Intra- and Extra-Amygdaloid Causal Connectivity.

Neuropsychopharmacology, 40:1782-1793.

Hampstead, B.M., Khoshnoodi, M. Yan, W., Deshpande, G. \& Sathian, K. (2015). Patterns of effective connectivity during memory encoding and retrieval differ between patients with mild cognitive impairment and healthy older adults. NeuroImage, in press, doi:10.1016/ j.neuroimage.2015.10.002 
Handwerker, D. A., Ollinger, J. M., \& D'Esposito, M. (2004). Variation of BOLD hemodynamic responses across subjects and brain regions and their effects on statistical analyses. Neuroimage, 21:1639-1651.

Havlicek, M., Friston, K. J., Jan, J., Brazdil, M., \& Calhoun, V. D. (2011). Dynamic modeling of neuronal responses in fMRI using cubature Kalman filtering. Neuroimage, $56: 2109-2128$.

Henriksson, L., Mur, M. \& Kriegeskorte, N. (2015). Faciotopy - a face-feature map with face-like topology in the human occipital face area. Cortex, 72:156-157.

Hutcheson, N. L., Sreenivasan, K. R., Deshpande, G., Reid, M. A., Hadley, J., White, D. M. et al. (2015). Effective connectivity during episodic memory retrieval in schizophrenia participants before and after antipsychotic medication. Human Brain Mapping, $36: 1442-1457$.

Kanwisher, N., McDermott, J. \& Chun, M.M. (1997). The fusiform face area: a module in human extrastriate cortex specialized for face perception. Journal of Neuroscience, 17:4302-4311.

Kapogiannis, D., Deshpande, G., Krueger, F., Thornburg, M. P., \& Grafman, J. H. (2014). Brain networks shaping religious belief. Brain Connectivity, 4:70-79.

Kasparian, K. (2013). Hemispheric differences in figurative language processing: contributions of neuroimaging methods and challenges in reconciling current empirical findings. Journal of Neurolinguistics, 26:1-21. 
Keller, S.S., Crow, T., Foundas, A., Amunts, K. \& Roberts, N. (2009). Broca's area: nomenclature, anatomy, typology and asymmetry. Brain \& Language, 109:29-48.

Kemmerer, D. \& Tranel, D. (2008). Searching for the elusive neural substrates of body part terms: a neuropsychological study. Cognitive Neuropsychology, 25:601-629.

Kucera, H. \& Francis, W.N. (1967). Computational Analysis of Present-day American English. Providence, RI: Brown University Press.

Lacey, S., Hagtvedt, H., Patrick, V.M., Anderson, A., Stilla, R., Deshpande, G., Hu, X., Sato, J.R., Reddy, S. \& Sathian, K. (2011a). Art for reward's sake: visual art recruits the ventral striatum. NeuroImage 55:420-433.

Lacey, S., Lin, J.B. \& Sathian, K. (2011b). Object and spatial imagery dimensions in visuohaptic representations. Experimental Brain Research, 213:267-273.

Lacey, S., Stilla, R. \& Sathian, K. (2012). Metaphorically feeling: comprehending textural metaphors activates somatosensory cortex. Brain \& Language, 120:416-421.

Lacey, S., Stilla, R., Sreenivasan, K., Deshpande, G. \& Sathian, K. (2014). Spatial imagery in haptic shape perception. Neuropsychologia, 60:144-158

Lakoff, G. \& Johnson, M. (1980). Metaphors We Live By. University of Chicago Press: Chicago [updated 2003].

Lambon Ralph, M.A., Sage, K., Jones, R.W. \& Mayberry, E.J. (2010a). Coherent concepts are computed in the anterior temporal lobes. Proceedings of the National Academy of Sciences USA, 107:2717-2722. 
Lambon Ralph, M.A., Cipolotti, L., Manes, F. \& Patterson, K. (2010b). Taking both sides: do unilateral anterior temporal lobe lesions disrupt semantic memory? Brain, 133:3243-3255.

Lambon Ralph, M.A., Ehsan, S., Baker, G.A. \& Rogers, T.T. (2012). Semantic memory is impaired in patients with unilateral anterior temporal lobe resection for temporal lobe epilepsy. Brain, 135:242-258.

Lee, S.S. \& Dapretto, M. (2006). Metaphorical vs. literal word meanings: fMRI evidence against a selective role of the right hemisphere. NeuroImage, 29:536-544.

Mariën, P., Ackermann, H., Adamaszek, M., Barwood, C.H.S., Beaton, A., et al. (2014). Consensus paper: language and the cerebellum: an ongoing enigma. Cerebellum, 13:386-410.

Motes, M.A., Malach, R. \& Kozhevnikov, M. (2008). Object-processing neural efficiency differentiates object from spatial visualizers. NeuroReport, 19:1727-1731.

Myers, A. \& Sowden, P.T. (2008). Your hand or mine? The extrastriate body area. NeuroImage, 42:1669-1677.

Nieuwenhuis, S., Forstmann, B.U. \& Wagenmakers, E.-J. (2015). Erroneous analyses of interactions in neuroscience: a problem of significance. Nature Neuroscience, 14:1105-1107.

Obert, A., Gierski, F., Calmus, A., Portefaix, C., Declercq, C. et al. (2014). Differential bilateral involvement of the parietal gyrus during predicative metaphor processing: an auditory fMRI study. Brain \& Language, 137:112-119.

Orlov, T., Makin, T.R. \& Zohary, E. (2010). Topographic representation of the human body in the occipitotemporal cortex. Neuron, 68:586-600. 
Price, A.R., Peelle, J.E., Bonner, M.F., Grossman, M. \& Hamilton, R.H. (2016). Causal evidence for a mechanism of semantic integration in the angular gyrus as revealed by highdefinition transcranial direct current stimulation. Journal of Neuroscience, 36:3829-3838.

Preusse, F., van der Meer, E., Deshpande, G., Krueger, F., \& Wartenburger, I. (2011). Fluid intelligence allows flexible recruitment of the parieto-frontal network in analogical reasoning. Frontiers in Human Neuroscience, 5:22.

Raczkowski, D., Kalat, J.W. \& Nebes, R. (1974). Reliability and validity of some handedness questionnaire items. Neuropsychologia, 12:43-47.

Ramachandran, V.S. \& Hubbard, E.M. (2001). Synesthesia - a window into perception, thought, and language. Journal of Consciousness Studies, 8:3-34.

Raposo, A., Moss, H.E., Stamatakis, E.A. \& Tyler, L.K. (2009). Modulation of motor and premotor cortices by actions, action words and action sentences. Neuropsychologia, 47:388-396.

Rapp, A.M., Leube, D.T., Erb, M., Grodd, W. \& Kircher, T.T.J. (2004). Neural correlates of metaphor processing. Cognitive Brain Research, 20:395-402.

Rapp, A.M., Mutschler, D.E. \& Erb, M. (2012). Where in the brain is non-literal language? A coordinate-based meta-analysis of functional magnetic resonance imaging studies. NeuroImage, 63:600-610.

Roebroeck, A., Formisano, E., \& Goebel, R. (2005). Mapping directed influence over the brain using Granger causality and fMRI. Neuroimage, 25:230-242. 
Sarubbo, S., De Benedictis, A., Maldonado, I.L., Basso, G. \& Duffau, H. (2013). Frontal terminations for the inferior fronto-occipital fascicle: anatomical dissection, DTI study and functional considerations on a multi-component bundle. Brain Structure \& Function, 218:21-37.

Sarubbo, S., De Benedictis, A., Merler, S., Mandonnet, E., Balbi, S. et al., (2015). Towards a functional atlas of human white matter. Human Brain Mapping, 36:3117-3136.

Sathian, K., Lacey, S., Stilla, R., Gibson, G. O., Deshpande, G., Hu, X. et al., (2011). Dual pathways for haptic and visual perception of spatial and texture information. Neuroimage, $57: 462-475$.

Sathian, K., Deshpande, G. \& Stilla, R. (2013). Neural changes with tactile learning reflect decision-level reweighting of perceptual readout. Journal of Neuroscience, 33:5387-5398.

Sreenivasan, K. R., Havlicek, M., \& Deshpande, G. (2015). Nonparametric hemodynamic deconvolution of FMRI using homomorphic filtering. IEEE Transactions in Medical Imaging, 34:1155-1163.

Stilla, R., Deshpande, G., LaConte, S., Hu, X., \& Sathian, K. (2007). Posteromedial parietal cortical activity and inputs predict tactile spatial acuity. Journal of Neuroscience, 27:11091-11102.

Stilla, R., Hanna, R., Hu, X., Mariola, E., Deshpande, G., \& Sathian, K. (2008). Neural processing underlying tactile microspatial discrimination in the blind: A functional magnetic resonance imaging study. Journal of Vision, 8(10):13, 1-19. 
Strenziok, M., Krueger, F., Deshpande, G., Lenroot, R. K., van der Meer, E., \& Grafman, J. (2010). Fronto-parietal regulation of media violence exposure in adolescents: a multimethod study. Social, Cognitive \& Affective Neuroscience, 6:537-547.

Talairach, J. \& Tournoux, P. (1998). Co-planar Stereotaxic Atlas of the Brain. Thieme Medical Publishers, New York.

Taylor, J.C., Wiggett, A.J. \& Downing, P.E. (2007). Functional MRI analysis of body and body part representations in the extrastriate and fusiform body areas. Journal of Neurophysiology, 98:1626-1633.

Toglia, M.P. \& Battig, W.F. (1978). Handbook of Semantic Word Norms. Hillsdale, NJ, USA: Lawrence Erlbaum Associates.

Van de Winckel, A., Sunaert, S., Wenderoth, N., Peeters, R., Van Hecke, P. et al., (2005). Passive somatosensory discrimination tasks in healthy volunteers: differential networks involved in familiar versus unfamiliar shape and length discrimination. NeuroImage, 26:441-453.

Vartanian, O. (2012). Dissociable neural systems for analogy and metaphor: implications for the neuroscience of creativity. British Journal of Psychology, 103:302-316.

Weiner, K.S. \& Grill-Spector, K. (2010). Sparsely-distributed organization of face and limb activations in human ventral temporal cortex. NeuroImage, 52:1559-1573.

Weiner, K.S., Grill-Spector, K. (2011). Not one extrastriate body area: using anatomical landmarks, hMT+, and visual field maps to parcellate limb-selective activations in human lateral occipitotemporal cortex. NeuroImage, 56:2183-2199. 
Weiner, K.S. \& Grill-Spector, K. (2013). Neural representations of faces and limbs neighbor in human high-level visual cortex: evidence for a new organization principle. Psychological Research, 77:74-97.

Weissenbacher, A., Kasess, C., Gerstl, F., Lanzenberger, R. et al. (2009). Correlations and anticorrelations in resting-state functional connectivity MRI: a quantitative comparison of preprocessing strategies. NeuroImage, 47:1408-1416.

Wen, X., Rangarajan, G., \& Ding, M. (2013). Is Granger Causality a Viable Technique for Analyzing fMRI Data? PloS ONE, 8:e67428.

Wheelock, M. D., Sreenivasan, K. R., Wood, K. H., Ver Hoef, L. W., Deshpande, G., \& Knight, D. C. (2014). Threat-related learning relies on distinct dorsal prefrontal cortex network connectivity. Neuroimage, 102 Pt 2, 904-912.

White, T., O’Leary, D., Magnotta, V., Arndt, S., Flaum, M. \& Andreasen, N.C. (2001). Anatomic and functional variability: The effects of filter size in group fMRI data analysis. NeuroImage, 13:577-588.

Wilson, M.D. (1988). The MRC Psycholinguistic Database: Machine Readable Dictionary, Version 2. Behavioral Research Methods \& Instruments, 20:6-11.

Xia, M., Wang, J., \& He, Y. (2013). BrainNet Viewer: A Network Visualization Tool for Human Brain Connectomics. PloS ONE, 8:e68910.

Yang, J. (2014). The role of the right hemisphere in metaphor comprehension: a metaanalysis of functional magnetic resonance imaging studies. Human Brain Mapping, $35: 107-122$. 
Yang, J. \& Shu, H. (2015). Involvement of the motor system in comprehension of non-literal action language: a meta-analysis study. Brain Topography, in press, doi: 10.1007/ s10548-015-0427-5 
Table 1 Analyses of metaphorical and control sentences, showing mean (SE) values of variables; results of ANOVAs comparing metaphorical and control sentences; and, for semantic variables, also results of t tests comparing obtained values for metaphorical sentences against mid-point of 7-point Likert scale: (a) lexical, (b) semantic, (c) acoustic.

\begin{tabular}{|c|c|}
\hline $\begin{array}{l}\text { Metaphoric } \\
\text { al }\end{array}$ & Control \\
\hline
\end{tabular}

(a) Lexical variables

$\begin{array}{lrrrr}\text { Syllables } & 6.3(.2) & 6.2(.2) & .4 & .53 \\ \text { Words } & 5.3(.1) & 4.5(.1) & 13.3 & <.001 \\ \text { Word frequency } & 115.0 & 134.5 & .4 & .56 \\ & (20.9) & (25.1) & & \\ \text { Imageability } & 5.7(.1) & 3.9(.1) & 128.6 & <.001\end{array}$

(b) Semantic

variables

$\begin{array}{lccccccc}\text { Interpretability } & 6.3(.1) & 6.8 & (.02) & 22.5 & <.001 & 28.1 & <.001 \\ \text { Naturalness } & 5.7(.1) & 6.6 & (.05) & 38.7 & <.001 & 14.9 & <.001 \\ \text { Figurativeness } & 5.8(.07) & 2.1 & (.05) & 2075.0 & <.001 & 35.5 & <.001 \\ \text { Familiarity } & 5.9(.1) & 6.6(.04) & 21.3 & <.001 & 15.7 & <.001 \\ \begin{array}{l}\text { Sentence } \\ \text { imageability }\end{array} & 5.9(.1) & 5.2 & (.08) & 26.8 & <.001 & 24.2 & <.001 \\ \text { Aptness } & 5.8(.1) & \mathrm{n} / \mathrm{a} & & & 23.5 & <.001 \\ \begin{array}{l}\text { Agreement with } \\ \text { control }\end{array} & 6.0(.07) & \mathrm{n} / \mathrm{a} & & & 35.7 & <.001\end{array}$

(c) Acoustic

variables

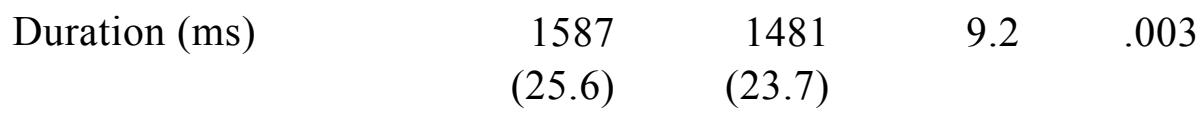


Speech rate

(syllables/s)

Pitch (Hz)

Intensity $(\mathrm{dB})$
$4.0(.1) \quad 4.2(.1)$

$131.9(.7)$

133.0

(1.2)
3.4

.07

.58

.45

$56.4 \quad(.2) \quad 56.7 \quad(.2)$
.63

.43 
Table 2 Analyses of literal and control sentences, mean (SE) values of variables and results of ANOVAs: (a) lexical, (b) semantic, (c) acoustic.

Literal

(a) Lexical variables

Syllables

Words

Word frequency

Imageability

(b) Semantic variables
$6.7(.04)$

$6.4(.07)$

$1.6(.05)$

$6.3(.05)$

$6.4(.06)$
Control

$4.8(.1)$

$4.1(.04)$

$105.2(16.2)$

$5.9(.06)$
$\mathrm{F}_{1,118}$

3.0

.09

.4

.5

$.04 \quad .8$

.05

.8

Naturalness

Figurativeness

Familiarity

Sentence imageability

(c) Acoustic variables

Duration (ms)

Speech rate (syllables/s)

Pitch $(\mathrm{Hz})$

Intensity $(\mathrm{dB})$
$1482(15.1) \quad 1476(15.5) \quad .1 \quad .8$

$3.1(.06) \quad 3.3(.07) \quad 3.3 \quad .1$

$134.0(1.6) \quad 132.1(.9) \quad 1.1$

$\begin{array}{lllll}56.2(.3) & 56.1 & (.3) & .2 & .7\end{array}$


Table 3 Examples of metaphorical and literal body-part sentences (body-part words in italics) with their respective control sentences.

\section{Control sentence}

\section{Metaphorical sentence}

He is under her thumb

He is under her control

He had to foot the bill

He had to pay the bill

He gave them a heads-up

He gave them a warning

\section{Literal sentence}

She scraped her knuckles

She scraped her plate

His feet were small

His chair was small

She closed her mouth

She closed her door 


\section{FIGURE LEGENDS}

Figure 1 Sequences of (a) stimulus generation and preparation and (b) studies.

Figure 2 (a) The interaction contrast of (metaphor $>$ control) $>$ (literal $>$ control) showing activation bilaterally in the STG extending into the STS, and on the left also extending posteriorly into the MTG at the location of the EBA; (b) Bonferroni-corrected (voxel-wise p $<.025)$ post-hoc contrast within a mask of the interaction result showed that, relative to their controls, metaphorical sentences activated bilateral STG and left EBA.

Figure 3 In participant-based analyses, the EBA metaphor-specific activity (blue) was also limb-selective, relative to face metaphors (see illustrative time-course curves), and shifted anterolaterally compared to the visual EBA (pink). The metaphor-specific focus was also active in item-wise analysis after filtering out nuisance acoustic variables (purple).

Figure 4 RSFC analyses showed an extensive network of areas (red; see Supplementary Table 1) connected to a seed region (blue) in the limb metaphor-specific EBA and areas in which resting-state activity correlated across participants with OSIVQ verbal scores (yellow) that partially overlapped (orange) with the seed-based network. Only left hemisphere shown; A, a: anterior; FG: fusiform gyrus; see main text for other abbreviations.

Figure 5 Effective connectivity analysis during metaphor processing. Note that the brain is viewed with the left hemisphere in the foreground and tilted toward the viewer (midline indicated for clarity); A, a: anterior; P: posterior; RH: right hemisphere; LH: left hemisphere; precun: precuneus; see main text for other abbreviations. 
Figure 1

\section{A Stimulus generation \& preparation}

Generate metaphorical and literal sentences relating to arm, leg, or face, and control sentences.

(Preliminary matching for number of words \& syllables, word frequency and imageability).

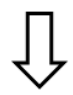

Assess interpretability of metaphorical sentences $(\mathrm{N}=10)$ and compare to their control sentences.

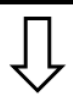

Final matching for number of words \& syllables, word frequency and imageability.

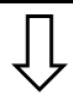

Semantic ratings $(\mathrm{N}=10)$ for interpretability, naturalness, figurativeness, familiarity, sentence imageability, aptness (metaphorical sentences only), \& agreement (metaphor-control pairs only).

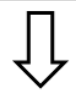

Recording of sentences and acoustic analysis of mean sentence duration $(\mathrm{ms})$, pitch $(\mathrm{Hz})$, \& intensity $(\mathrm{dB})$.

B Study sequence

Scan session 1: language tasks

(i) Resting-state functional connectivity (1 run)

(ii) Metaphorical \& control sentences (2 runs)

(iii) (Break)

(iv) Literal \& control sentences (2 runs)

Scan session 2: localizer tasks

Scan session 3: localizer tasks

(i) Motor: primary motor cortex (4 runs)

(ii) Participants also complete OSIVQ

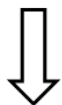

Post-scan : rating tasks

Participants rate metaphorical \& literal sentences for familiarity \& imageability 
Figure 2
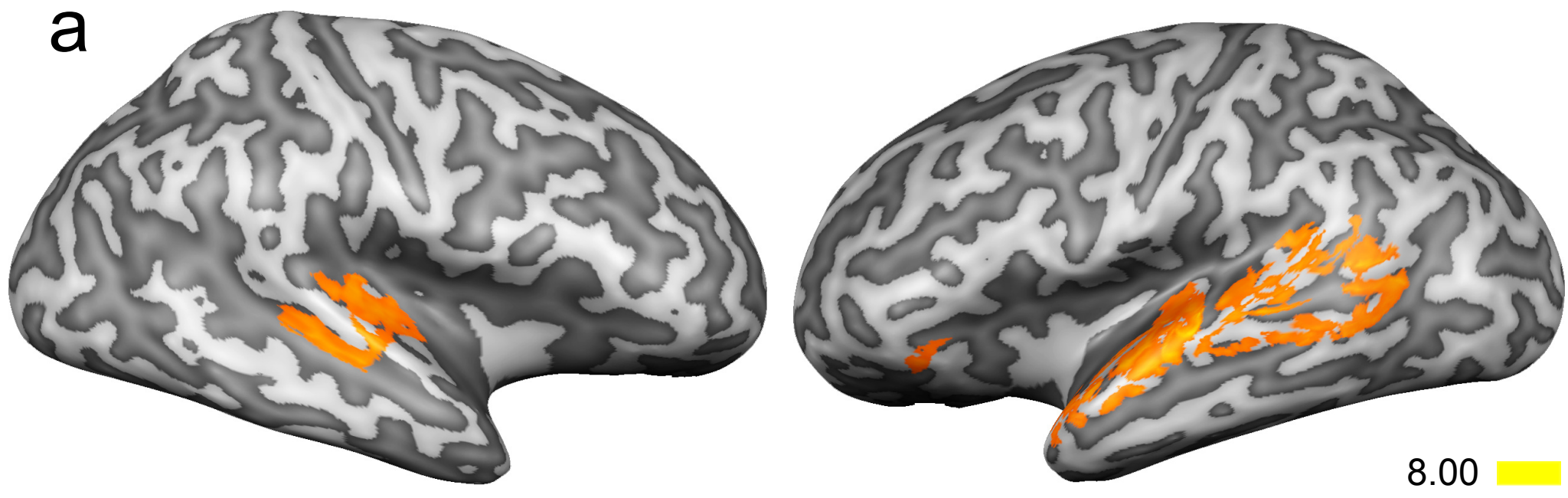

$\mathrm{RH}$

$\mathrm{LH}$

2.20
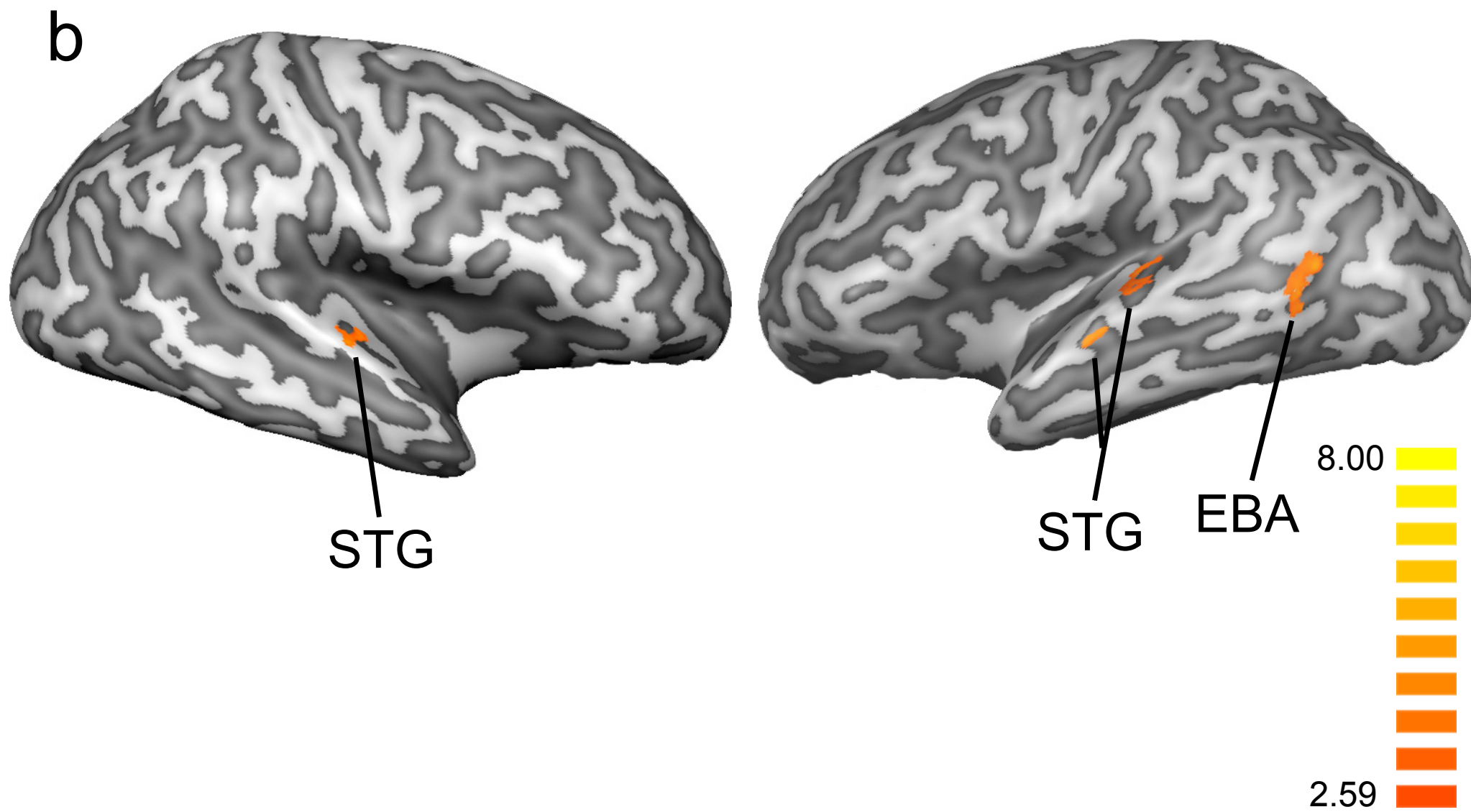
Figure 3

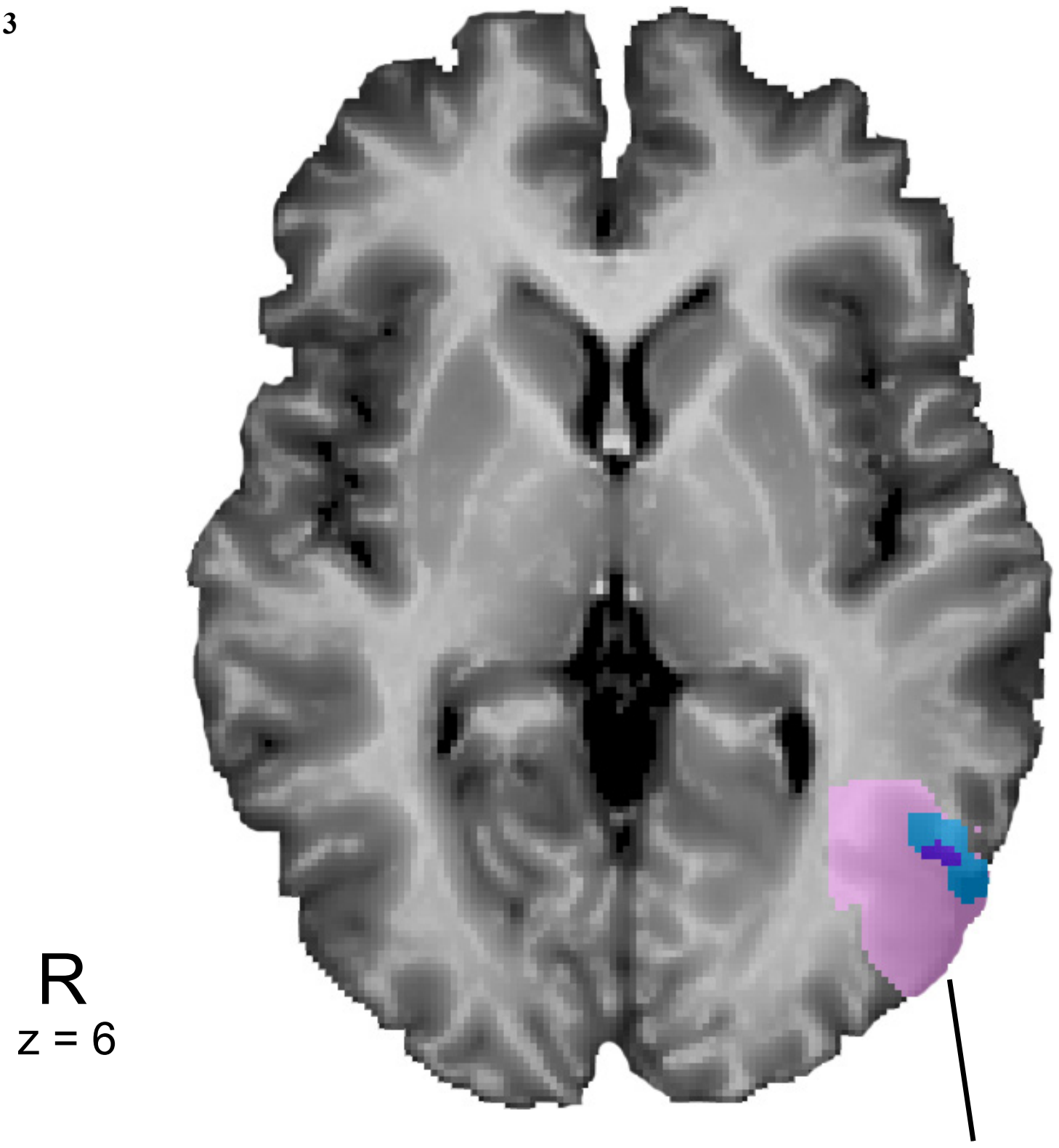

EBA

limb metaphor

limb control

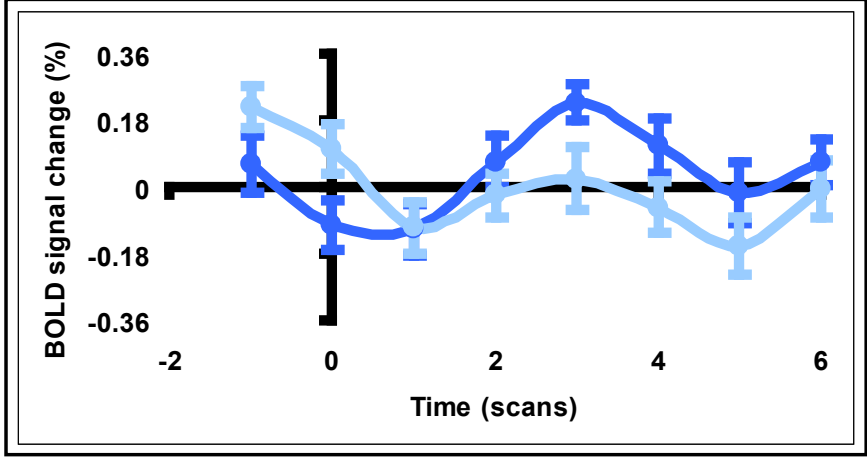

face metaphor

face control

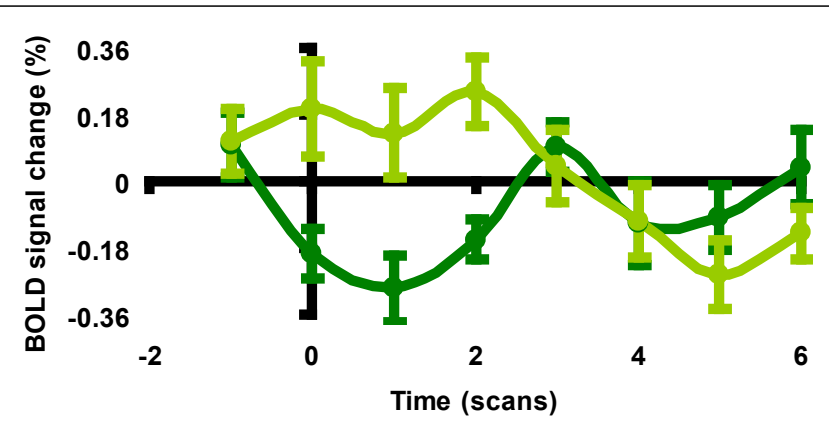


Figure 4
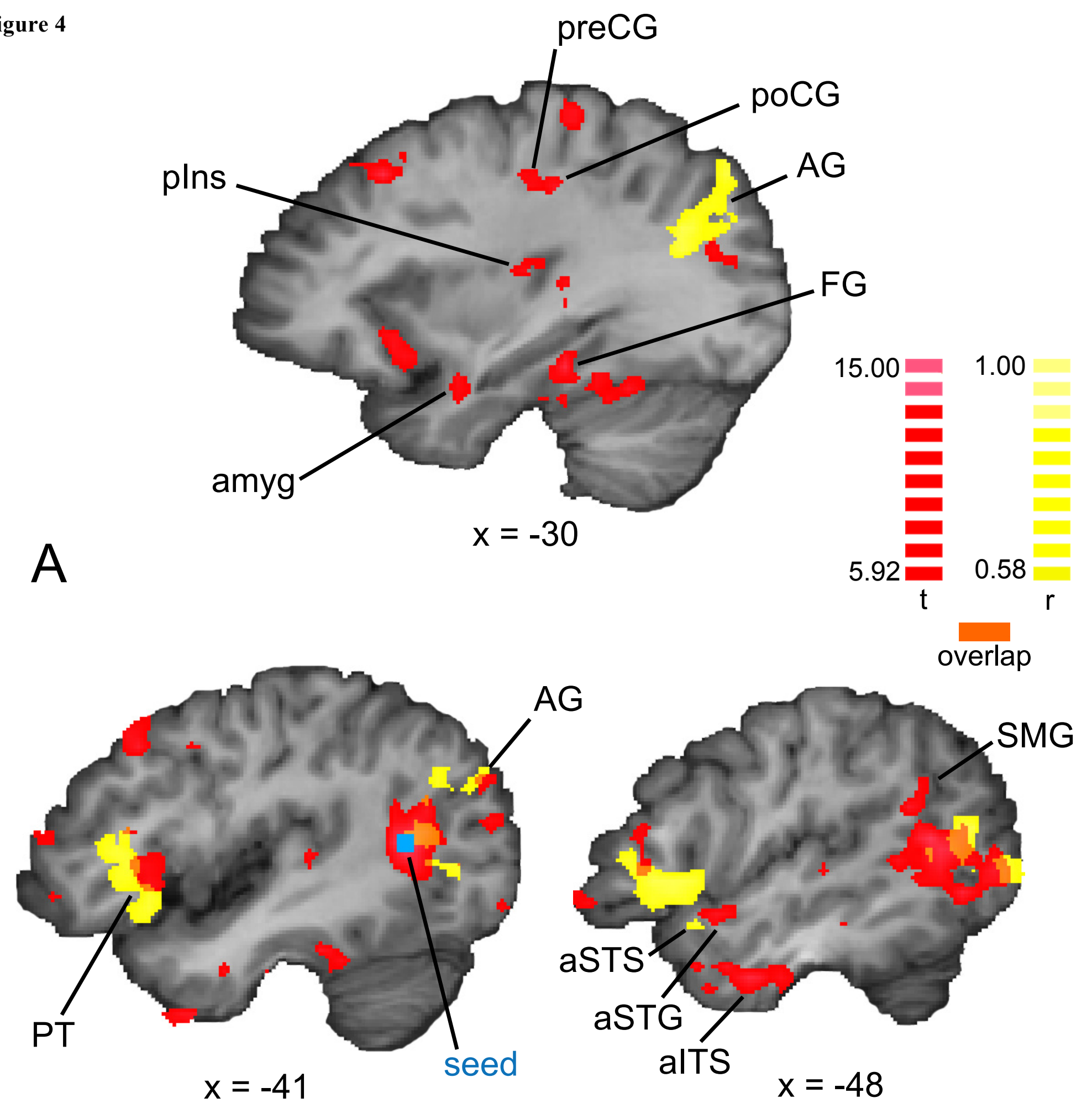

overlap 
Figure 5

\section{$\mathrm{RH}$}

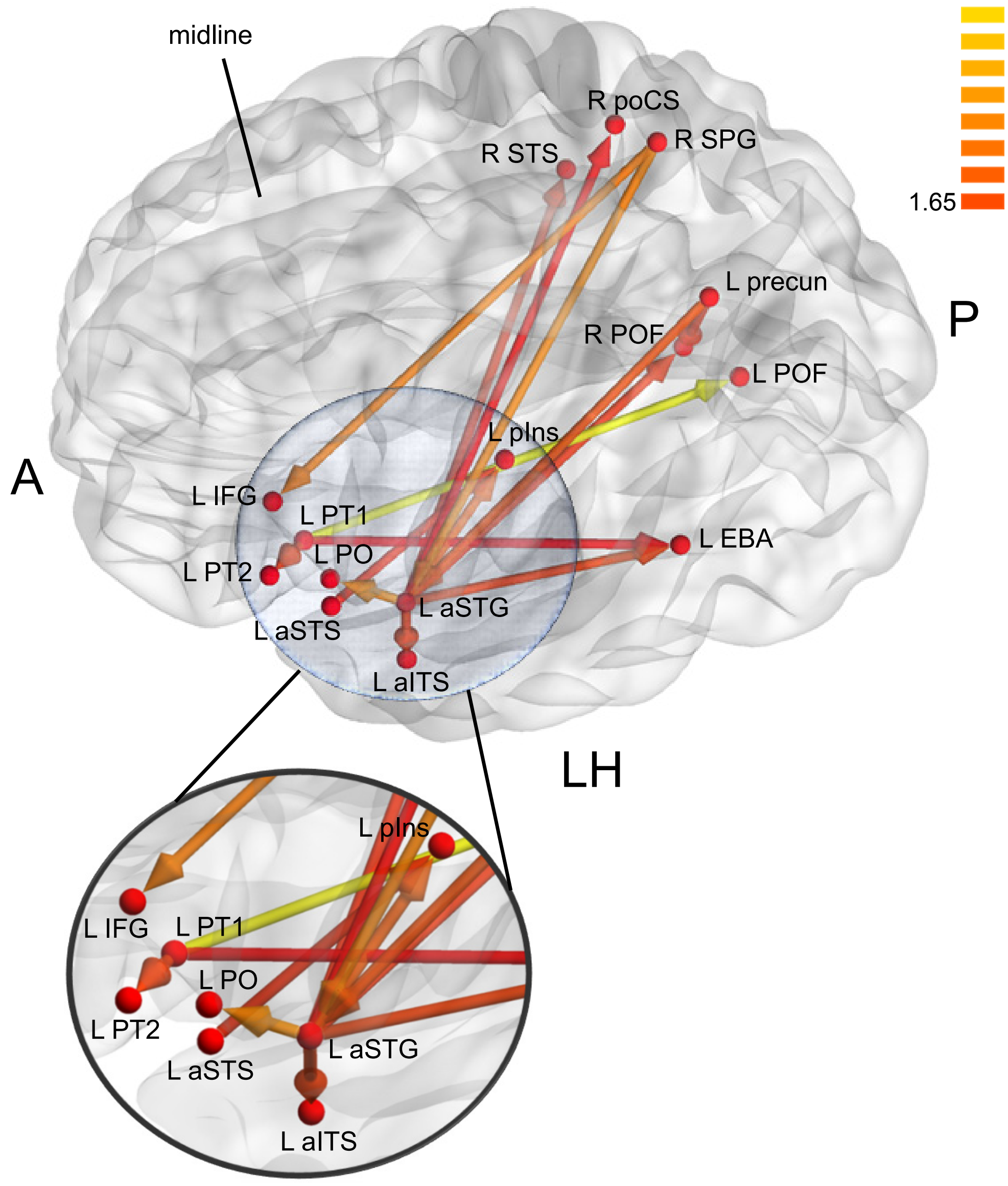

